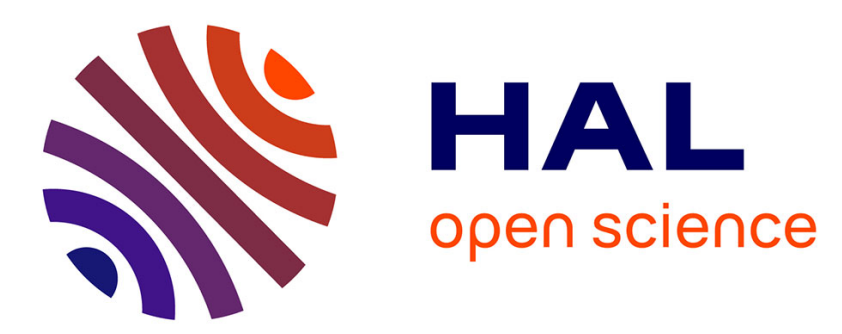

\title{
Geometric Approach to b-Symbol Hamming Weights of Cyclic Codes
}

Minjia Shi, Ferruh Ozbudak, Patrick Solé

\section{To cite this version:}

Minjia Shi, Ferruh Ozbudak, Patrick Solé. Geometric Approach to b-Symbol Hamming Weights of Cyclic Codes. IEEE Transactions on Information Theory, 2021, pp.1 - 1. 10.1109/tit.2021.3069772 . hal-03189478

\section{HAL Id: hal-03189478 \\ https://hal.science/hal-03189478}

Submitted on 3 Apr 2021

HAL is a multi-disciplinary open access archive for the deposit and dissemination of scientific research documents, whether they are published or not. The documents may come from teaching and research institutions in France or abroad, or from public or private research centers.
L'archive ouverte pluridisciplinaire HAL, est destinée au dépôt et à la diffusion de documents scientifiques de niveau recherche, publiés ou non, émanant des établissements d'enseignement et de recherche français ou étrangers, des laboratoires publics ou privés. 


\title{
Geometric Approach to $b$-Symbol Hamming Weights of Cyclic Codes
}

\author{
Minjia Shi ${ }^{(}$, Ferruh Özbudak ${ }^{(}$, and Patrick Solé
}

\begin{abstract}
Symbol-pair codes were introduced by Cassuto and Blaum in 2010 to protect pair errors in symbol-pair read channels. Recently Yaakobi, Bruck and Siegel (2016) generalized this notion to $b$-symbol codes in order to consider consecutive $b$ errors for a prescribed integer $b \geq 2$, and they gave constructions and decoding algorithms. Cyclic codes were considered by various authors as candidates for symbol-pair codes and they established minimum distance bounds on (certain) cyclic codes. In this paper we use algebraic curves over finite fields in order to obtain tight lower and upper bounds on $b$-symbol Hamming weights of arbitrary cyclic codes over $\mathbb{F}_{q}$. Here $b \geq 2$ is an arbitrary prescribed positive integer and $\mathbb{F}_{q}$ is an arbitrary finite field. We also present a stability theorem for an arbitrary cyclic code $C$ of dimension $k$ and length $n$ : the $b$-symbol Hamming weight enumerator of $C$ is the same as the $k$-symbol Hamming weight enumerator of $C$ if $k \leq b \leq n-1$. Moreover, we give improved tight lower and upper bounds on $b$-symbol Hamming weights of some cyclic codes related to irreducible cyclic codes. Throughout the paper the length $n$ is coprime to $q$.
\end{abstract}

Index Terms-Cyclic code, $b$-symbol error, algebraic curve, Weil-Serre bound, irreducible cyclic code.

\section{INTRODUCTION}

$\mathbf{S}$ YMBOL-PAIR codes were introduced by Cassuto and Blaum [2], [3] to combat symbol-pair errors in symbol-pair channels. This model was used to address channels with high write resolution but low read resolution, so that individual symbols cannot be read off due to physical limitations. In this new model the errors are no longer individual symbol errors, but rather symbol-error pair errors, where in a symbol-pair error at least one of the symbols is erroneous.

The seminal works [2]-[4] established relationships between the minimum Hamming distance of an error correcting code

Manuscript received September 14, 2019; revised October 31, 2020; accepted March 13, 2021. This work was supported in part by the National Natural Science Foundation of China under Grant 12071001 and Grant 61672036, in part by the Excellent Youth Foundation of Natural Science Foundation of Anhui Province under Grant 1808085J20, in part by the Academic Fund for Outstanding Talents in Universities under Grant gxbjZD03, and in part by the Natural Science Foundation of Anhui Province under Grant 2008085QA04. (Corresponding author: Minjia Shi.)

Minjia Shi is with the Key Laboratory of Intelligent Computing Signal Processing, Ministry of Education, School of Mathematical Sciences, Anhui University, Hefei 230601, China (e-mail: smjwcl.good@163.com).

Ferruh Özbudak is with the Department of Mathematics, Institute of Applied Mathematics, Middle East Technical University, 06800 Ankara, Turkey (e-mail: ozbudak@metu.edu.tr).

Patrick Solé is with I2M, Aix Marseille University, Centrale Marseille, CNRS, 13007_ Marseille, France (e-mail: sole@enst.fr).

Communicated by S. Ghorede Associate Editor for Coding Theory.

Digital Object Identifier 10.1109/TIT.2021.3069772 and the minimum pair distance, constructed some codes for pair distance and gave decoding algorithms.

The minimum pair distance of linear cyclic codes has been studied by Cassuto and Blaum [3], Kai et al. [13], and recently by Yaakobi et al. [19]. In particular, Yaakobi et al. obtained an elegant result on the pair distance of binary cyclic codes of dimension at least 2: $d_{2}(C) \geq d_{1}(C)+\left\lceil\frac{d_{1}(C)}{2}\right\rceil \simeq \frac{3}{2} d_{1}(C)$, where $d_{2}(C)$ is the minimum pair distance of $C$ and $d_{1}(C)$ is the minimum (Hamming) distance of $C$. Moreover, in [19] they considered the more general problem of consecutive $b$-symbol errors instead of only 2 -symbol errors for a prescribed integer $b \geq 2$. They generalized some results of $b=2$ to the case of $b \geq 2$.

Let $\mathbb{F}_{q}$ be an arbitrary finite field. In this paper we use algebraic curves over finite fields (equivalently algebraic function fields over finite fields) in order to study lower and upper bounds on an arbitrary cyclic code $C$ over $\mathbb{F}_{q}$ of length $n$, where $b$ is a prefixed integer such that $2 \leq b \leq n-1$. Our main contributions are:

- We obtain tight lower and upper bounds for $b$-symbol Hamming weights of arbitrary cyclic codes.

- We give a stability theorem for $b$-symbol Hamming weights: if $C$ is an arbitrary cyclic code of length $n$ and dimension $k$, then for any integer $b$ in the range $k \leq b \leq n-1$ the $b$-symbol Hamming weight enumerator of $C$ is the same as the $k$-symbol Hamming weight enumerator of $C$.

- We obtain improved lower and upper bounds for $b$-symbol Hamming weights of some cyclic codes related to irreducible cyclic codes.

We also find a connection between maximal and minimal curves over finite fields and the lower and upper bounds of $b$-symbol Hamming weights of arbitrary cyclic codes. Using this connection and inspired by the important result $d_{2}(C) \geq$ $\frac{3}{2} d_{1}(C)$ of Yaakobi et al. [19, Theorem 1], we obtain further inequalities between $d_{b+\delta}(C)$ and $d_{b}(C)$ for some cyclic codes $C$.

For any code $C$ of length $n$ over $\mathbb{F}_{q}$, there is a canonical code $C^{(b)}$ of length $n$ over the alphabet $\mathbb{F}_{q}^{b}$ such that the $b$-symbol Hamming weight enumerator of $C$ is the same as the Hamming weight enumerator of $C$. This follows naturally from the definition by an explicit $\mathbb{F}_{q}$-linear map $\pi_{b}$. We could not find this map in the literature and we explain it in Section 2 below.

The rest of the paper is organized as follows: We give some preliminaries and further notation in Section 2. We present a 
trace representation of $C^{(b)}$ in Section 3, that we use in our proofs. We specialize to a subclass of cyclic codes related to irreducible cyclic codes in Section 4. This allows us to present some of our methods in detail and we also get improved bounds on the $b$-symbol weights in this subclass. We give our results for arbitrary cyclic codes in Section 5. We also have an appendix providing background material on algebraic function fields that we use in Section 4 and 5. We conclude in Section 6.

\section{Preliminaries}

We start by fixing a part of our notation:

- $\mathbb{F}_{q}$ : finite field with $q$ elements.

- $n \geq 3$ : an integer with $\operatorname{gcd}(n, q)=1$.

- $2 \leq b \leq n-1$ : an integer.

- For a finite set $A$, let $|A|$ denote its cardinality.

- $C$ : an $\mathbb{F}_{q}$-linear subspace of $\mathbb{F}_{q}^{n}$. We assume that $|C|>1$ omitting the trivial case. $C$ is called a linear code of length $n$ over $\mathbb{F}_{q}$. We also refer $C$ just as code throughout this paper. Elements of $C$ are called codewords of $C$.

\section{- $k=\operatorname{dim}_{\mathbb{F}_{q}} C$.}

We present further notation and preliminaries in the following subsections.

\section{A. Hamming Weight, Hamming Distance and Hamming Weight Enumerator}

Let $\mathbb{A}$ be a nonempty finite set, which stands for the alphabet to be fixed. Throughout the paper $\mathbb{A}$ becomes $\mathbb{F}_{q}$ or $\mathbb{F}_{q}^{b}=\underbrace{\mathbb{F}_{q} \times \cdots \times \mathbb{F}_{q}}$. Let $\alpha=\left(\alpha_{1}, \ldots, \alpha_{n}\right) \in \mathbb{A}^{n}$ and

\section{$b$ times}

$\beta=\left(\beta_{1}, \ldots, \beta_{n}\right) \in \mathbb{A}^{n}$. The Hamming weight $\|\alpha\|$ of $\alpha$ is the nonnegative integer

$$
\|\alpha\|=\left|\left\{1 \leq i \leq n: \alpha_{i} \neq 0\right\}\right| .
$$

The Hamming distance $d(\alpha, \beta)$ between $\alpha$ and $\beta$ is the nonnegative integer

$$
d(\alpha, \beta)=\left|\left\{1 \leq i \leq n: \alpha_{i} \neq \beta_{i}\right\}\right| .
$$

Let $\mathcal{C} \subseteq \mathbb{A}^{n}$ be a subset with $|\mathcal{C}| \geq 2$. The minimum Hamming distance $d(\mathcal{C})$ of $\mathcal{C}$ is the integer

$$
d(\mathcal{C})=\min \{d(\alpha, \beta): \alpha, \beta \in \mathcal{C} \text { and } \alpha \neq \beta\} .
$$

If $\mathcal{C}$ is further closed under addition, then it is well known and easy to observe that

$$
d(\mathcal{C})=\min \{\|\alpha\|: \alpha \neq \mathbf{0}\} .
$$

Assume that $\mathcal{C} \subseteq \mathbb{A}^{n}$ is a subset which is closed under addition. For $0 \leq i \leq n$, let $A_{i}$ be the nonnegative integer

$$
A_{i}=|\{\alpha \in \mathcal{C}:\|\alpha\|=i\}| .
$$

The polynomial $A(Z)=A_{0}+A_{1} Z+\cdots+A_{n} Z^{n} \in \mathbb{Z}[Z]$ with these nonnegative integer coefficients is called the Hamming weight enumerator of $\mathcal{C}$.
B. b-Symbol Hamming Weight, b-Symbol Hamming Minimum Distance and b-Symbol Hamming Weight Enumerator

Recall that $b$ is an integer with $2 \leq b \leq n-1$. Let $\pi_{b}$ : $\mathbb{F}_{q}^{n} \rightarrow\left(\mathbb{F}_{q}^{b}\right)^{n}$ the map

$$
\begin{aligned}
\left(\alpha_{0}, \ldots, \alpha_{i}, \ldots, \alpha_{n-1}\right) \mapsto & \left(\left(\alpha_{0}, \alpha_{1}, \ldots, \alpha_{b-1}\right), \ldots,\right. \\
& \left(\alpha_{i}, \alpha_{i+1}, \ldots, \alpha_{i+b-1}\right), \ldots, \\
& \left.\left(\alpha_{n-1}, \alpha_{0}, \ldots, \alpha_{n+b-1}\right)\right),
\end{aligned}
$$

where the indices are modulo $n$, It is clear that $\pi_{b}$ is an $\mathbb{F}_{q}$-linear map.

Example 1: For $q=2, n=4, b=3$ and $\alpha=$ $(0,1,1,0,0) \in \mathbb{F}_{2}^{5}$ we have

$$
\begin{aligned}
\pi_{3}(\alpha) & =((0,1,1),(1,1,0),(1,0,0),(0,0,0),(0,0,1)) \\
& \in\left(\mathbb{F}_{2} \times \mathbb{F}_{2} \times \mathbb{F}_{2}\right)^{5} .
\end{aligned}
$$

The Hamming weight of $\pi_{3}(\alpha)$ over the alphabet $\mathbb{A}=\mathbb{F}_{2} \times$ $\mathbb{F}_{2} \times \mathbb{F}_{2}$ is $1+1+1+0+1=4$ (see Subsection II-A).

Put $\mathbb{A}=\underbrace{\mathbb{F}_{q} \times \cdots \times \mathbb{F}_{q}}_{b \text { times }}$. Recall that $C \subseteq \mathbb{F}_{q}^{n}$ is a linear code of length $n$ over $\mathbb{F}_{q}$. Let $C^{(b)}=\pi_{b}(C) \subseteq \mathbb{A}^{n}$ be the image of $C$ under the $\mathbb{F}_{q}$-linear map $\pi_{b}$. Note that $C^{(b)}$ is closed under addition. Using the notation of Subsection II-A, the Hamming weight minimum distance of $C^{(b)}$ and the Hamming weight enumerator of $C^{(b)}$ are well defined. The Hamming weight minimum distance of $C^{(b)}$ is called the b-symbol Hamming minimum distance of $C$. The Hamming weight enumerator of $C^{(b)}$ is called the b-symbol Hamming weight enumerator of $C$. Similarly for a codeword $c \in C$, the Hamming weight of $\pi_{b}(c) \in \mathbb{A}^{n}$ is called the b-symbol Hamming weight of $c$.

We also denote $C$ as $C^{(1)}$.

\section{Cyclic Code of Length $n$ Over $\mathbb{F}_{q}$ and Its Nonzero Set}

We further fix and assume the following from now on throughout the paper:

- $r \geq 2$ : an integer such that $n \mid\left(q^{r}-1\right)$.

- $\eta \in \mathbb{F}_{q^{r}}^{*}$ : a primitive $n$-th root of 1 .

- $C$ : an arbitrary (if not stated otherwise) cyclic code of length $n$ over $\mathbb{F}_{q}$.

The existence of $r$ follows by the assumption that $\operatorname{gcd}(n, q)=1$.

We need to introduce some basic facts on cyclic codes. We refer, for example [15], for the details. It is possible to identify an element $\left(a_{0}, a_{1}, \ldots, a_{n-1}\right) \in \mathbb{F}_{q}^{n}$ with the polynomial $a_{0}+a_{1} z+\cdots+a_{n-1} z^{n-1} \in \mathbb{F}_{q}[z]$. Let $R$ be the quotient ring of $\mathbb{F}_{q}[z]$ given by $R=\mathbb{F}_{q}[z] /\left\langle z^{n}-1\right\rangle$. Using this identification, cyclic codes of length $n$ over $\mathbb{F}_{q}$ are exactly ideals of $R$.

Let $I$ be the ideal of $R$ corresponding to $C$. It is well known that the ideals of $R$ are principal. Hence there exists a uniquely determined monic polynomial $g(z) \in \mathbb{F}_{q}[z]$ of smallest degree such that $g(z)+<z^{n}-1>\in I$. This polynomial is called the generator polynomial of $C$. Recall that $k$ is the dimension of $C$ over $\mathbb{F}_{q}$. It is well known that $\operatorname{deg} g(z)=n-k$ and $g(z) \mid\left(z^{n}-1\right)$ in the polynomial ring $\mathbb{F}_{q}[z]$.
140 141 142 143 144 
As $n \mid\left(q^{r}-1\right)$, there is no repeated root of $g(z)$ and $g(z)$ splits into its linear factors over $\mathbb{F}_{q^{r}}$. Let $S \subseteq\{0,1, \ldots, n-1\}$ be the subset such that the roots of $g(z)$ are exactly $\left\{\eta^{i}\right.$ : $i \in S\}$. Let $\tilde{S}$ be the complement, i.e. $\tilde{S}=\{0,1, \ldots, n-1\} \backslash S$. Let $U \subseteq\{0,1, \ldots, n-1\}$ be the subset of cardinality $k$ defined as $U=\{-j \bmod n: j \in \tilde{S}\}$. We call $U$ the nonzero set of $C$.

Example 2: Let $q=4, n=21$ and $r=3$. Let $\eta \in \mathbb{F}_{4^{3}}^{*}$ be a primitive 21-th root of 1 . We choose $\eta$ as a root of $x^{6}+$ $x^{5}+x^{4}+x^{2}+1 \in \mathbb{F}_{2}[x]$. Let

$$
\begin{aligned}
g(z)= & \left(z-\eta^{9}\right)\left(z-\eta^{15}\right)\left(z-\eta^{18}\right)\left(z-\eta^{5}\right) \\
& \left(z-\eta^{20}\right)\left(z-\eta^{17}\right)\left(z-\eta^{10}\right)\left(z-\eta^{19}\right) \\
& \left(z-\eta^{13}\right)\left(z-\eta^{7}\right) .
\end{aligned}
$$

It turn out that $g(z) \in \mathbb{F}_{4}[z]$. Namely we have

$$
\begin{aligned}
g(z)= & z^{10}+\theta z^{9}+\theta z^{8}+\theta^{2} z^{7}+z^{6}+\theta z^{5} \\
& +\theta^{2} z^{4}+z^{2}+\theta z+\theta^{2},
\end{aligned}
$$

where $\theta \in \mathbb{F}_{4}$ with $\theta^{2}+\theta+1=0$. It is clear that $g(z) \mid$ $\left(z^{21}-1\right)$ over $\mathbb{F}_{4}$. Let $C$ be the cyclic code of length 21 over $\mathbb{F}_{4}$ generated by $g(z)$. Under notation above we have

$$
\begin{aligned}
& S=\{5,7,9,10,13,15,17,18,19,20\}, \\
& \tilde{S}=\{0,1,2,3,4,6,8,11,12,14,16\}
\end{aligned}
$$

and hence the nonzero set $U$ of $C$ is given by

$$
U=\{0,5,7,9,10,13,15,17,18,19,20\} .
$$

We also fix the following from now on throughout the paper:

- $U \subseteq\{0,1, \ldots, n-1\}$ : the nonzero set of $C$.

Note that $U$ and $C$ determine each other uniquely.

\section{Trace Representation of a Cyclic Code}

In this subsection we present a trace representation of $C$. We use well known methods, see for example, [16, Chapter 9] and the references therein.

The cyclic group $\operatorname{Gal}\left(\mathbb{F}_{q^{r}} / \mathbb{F}_{q}\right)$ is generated by the Frobenius automorphism $x \mapsto x^{q}$. There is an action of $\operatorname{Gal}\left(\mathbb{F}_{q^{r}} / \mathbb{F}_{q}\right)$ on $\{0,1, \ldots, n-1\}$. The action of the Frobenius automorphism is given as follows: $u \in\{0,1, \ldots, n-1\} \mapsto u q$ $\bmod n \in\{0,1, \ldots, n-1\}$. For any integer $u \in\{0,1, \ldots$, $n-1\}$, the orbit $\left\{u^{i} \bmod n \in\{0,1, \ldots, n-1\}: 0 \leq i \leq\right.$ $r-1\}$ of $u$ under this action is called the $q$-cyclotomic coset of $u$ modulo $n$. A subset $A \subseteq\{0,1, \ldots, n-1\}$ is called closed if $u \in A$ implies that $u q \bmod n \in A$. A closed set is a disjoint union of $q$-cyclotomic cosets modulo $n$.

Recall that $U$ is the nonzero set of the cyclic code $C$. It is well known that $U$ is a closed set and hence hence $U$ is a disjoint union of $q$-cyclotomic cosets modulo $n$. Note that the disjoint decomposition of $U$ into its disjoint subsets, which are $q$-cyclotomic cosets modulo $n$, is uniquely determined. Let $U_{0}$ be a subset of $U$ such that there is exactly one element in $U_{0}$ for each $q$-cyclotomic coset modulo $n$ in this disjoint decomposition of $U$. Note that $U_{0}$ is not uniquely determined in general. We call that $U_{0}$ is a basic nonzero set of $C$.

We further fix the following from now on throughout the paper:
- $\operatorname{Tr}: \mathbb{F}_{q^{r}} \rightarrow \mathbb{F}_{q}$ : the trace map defined as $x \mapsto x+x^{q}+{ }_{223}$ $\cdots+x^{q^{r-1}}$.

Note that $\operatorname{Tr}$ is a surjective and $\mathbb{F}_{q}$-linear map. subspace of $\mathbb{F}_{q^{r}}[x]$ defined as

$$
P\left(U_{0}\right)=\left\{a_{1} x^{u_{1}}+\cdots+a_{\rho} x^{u_{\rho}}: a_{1}, \ldots, a_{\rho} \in \mathbb{F}_{q^{r}}\right\} . \quad 228
$$

For $f(x) \in P\left(U_{0}\right)$, we use the short notation $\operatorname{Tr}(f(x))$ for the $n$-tuple

$$
\underline{\operatorname{Tr}(f(x))}=\left(\operatorname{Tr}\left(f\left(\eta^{0}\right)\right), \cdots, \operatorname{Tr}\left(f\left(\eta^{n-1}\right)\right) \in \mathbb{F}_{q}^{n} .\right.
$$

It is well known that we have a trace representation for $C$ given by

$$
C=\left\{\underline{\operatorname{Tr}(f(x))}: f \in P\left(U_{0}\right)\right\},
$$

where we are free to choose an arbitrary basic nonzero set $U_{0}$ of $C$. Namely, a generic element $c=\left(c_{0}, c_{1}, \ldots, c_{n-1}\right)$ of $C$ is given by

$$
c=\underline{\operatorname{Tr}(f(x))} \in \mathbb{F}_{q}^{n} \text { and } f \in P\left(U_{0}\right) .
$$

Hence for $f \in P\left(U_{0}\right)$, we also use the notation $c(f)$ to donate the codeword

$$
c(f)=\left(\operatorname{Tr}(f(1)), \operatorname{Tr}(f(\eta)), \ldots, \operatorname{Tr}\left(f\left(\eta^{n-1}\right)\right)\right)
$$

of $C$.

Example 3: Let $q=4, n=21$ and $r=3$. We keep the notation of Example 2. Hence $\eta \in \mathbb{F}_{4^{3}}^{*}$ is a primitive 21-th root of 1 as in Example 2.

All 4-cyclotomic cosets modulo 21 are as follows:

$$
\begin{aligned}
& \overline{0}=\{0\}, \overline{1}=\{1,4,16\}, \overline{2}=\{2,8,11\}, \\
& \overline{3}=\{3,12,6\}, \overline{5}=\{5,20,17\}, \overline{7}=\{7\}, \\
& \overline{9}=\{9,15,18\}, \overline{10}=\{10,19,13\}, \overline{14}=\{14\} .
\end{aligned}
$$

Let $C$ be the cyclic code of length 21 over $\mathbb{F}_{4}$ defined in Example 2. We observe that the nonzero set $U$ of $C$ is a disjoint union of 4 -cyclotomic cosets modulo 21 given by

$$
\begin{aligned}
U= & \{0\} \sqcup\{5,10,17\} \sqcup\{7\} \sqcup\{9,15,18\} \\
& \sqcup\{10,19,13\},
\end{aligned}
$$

where $\sqcup$ indicates that the subsets $\{0\}, \ldots,\{10,19,13\}$ are pairwise disjoint. Hence a basic nonzero set $U_{0}$ of $C$ is

$$
U_{0}=\{0,5,7,9,10\} \text {. }
$$

For an arbitrary codeword $\mathbf{c}=\left(c_{0}, c_{1}, \ldots, c_{20}\right)$ of $C \subseteq \mathbb{F}_{4}^{21}$, there exists $f(x) \in P\left(U_{0}\right)=\left\{a_{0}+a_{5} x^{5}+a_{7} x^{7}+a_{9} x^{9}+\right.$ $\left.a_{10} x^{10}: a_{0}, a_{5}, a_{7}, a_{9}, a_{10} \in \mathbb{F}_{4^{3}}\right\}$ such that $\mathbf{c}=c(f)$. Namely there exist $a_{0}, a_{5}, a_{7}, a_{9}, a_{10} \in \mathbb{F}_{4^{3}}$ such that

$$
c_{i}=\operatorname{Tr}\left(a_{0}+a_{5} \eta^{5 i}+a_{7} \eta^{7 i}+a_{9} \eta^{9 i}+a_{10} \eta^{10 i}\right)
$$

$$
224
$$




\section{Trace Representations of $C^{(b)}$}

Note that the $b$-symbol Hamming weights of codewords of $C$ are defined in terms of the Hamming weights of the codewords of $C^{(b)}$ over the alphabets $\mathbb{F}_{q}^{b}$. We refer to Subsection II-B for a definition of the code $C^{(b)}$.

In this section we present a trace representation of $C^{(b)}$, where $C$ is an arbitrary cyclic code over $\mathbb{F}_{q}$ of length coprime to $q$. This section is one of the contributions of this paper as we could not find such an approach for the $b$-symbol Hamming weights of codewords of $C$ in the literature.

First we need a definition.

Definition III.1: For any integer $0 \leq t \leq n-1$ and $f \in$ $P\left(U_{0}\right)$, let $f^{(t)}$ denote the polynomial in $P\left(U_{0}\right)$ given by

$$
f^{(t)}(x)=f\left(\eta^{t} x\right) .
$$

We give our trace representation in the next theorem. We will use this representation in our proofs.

Theorem III.2: Let $C$ be an arbitrary cyclic code over $\mathbb{F}_{q}$ of length coprime to $q$. Let $2 \leq b \leq n-1$ be an integer. For the code $C^{(b)}$ of length $\mathrm{n}$ over the alphabet $\mathbb{F}_{q}^{b}$ we have

$C^{(b)}=\left\{\left(\underline{\operatorname{Tr}(f(\eta))}, \cdots, \underline{\operatorname{Tr}\left(f^{(b-1)}(\eta)\right)}\right): f \in P\left(U_{0}\right)\right\}$.

A generic element $\left(\beta_{0}, \ldots, \beta_{n-1}\right)$ of $C^{(b)}$ is given by

$\beta_{i}=\left(\operatorname{Tr}\left(f\left(\eta^{i}\right)\right), \operatorname{Tr}\left(f^{(1)}\left(\eta^{i}\right)\right), \ldots, \operatorname{Tr}\left(f^{(b-1)}\left(\eta^{i}\right)\right)\right) \in \mathbb{F}_{q}^{b}$

$f \in P\left(U_{0}\right), 0 \leq i \leq n-1$.

Proof: Let $f \in P\left(U_{0}\right)$ and $c(f) \in C$ be the corresponding codeword of the cyclic code $C$. We have

$$
\begin{aligned}
c(f) & =\left(c_{0}, c_{1}, \ldots, c_{n-1}\right) \\
& =\left(\operatorname{Tr}\left(f\left(\eta^{0}\right)\right), \operatorname{Tr}\left(f\left(\eta^{1}\right)\right), \ldots, \operatorname{Tr}\left(f\left(\eta^{n-1}\right)\right)\right) .
\end{aligned}
$$

Let $c^{(b)}(f)=\pi_{b}(c(f))$ be the corresponding codeword of $C^{(b)}$. Putting $c^{(b)}(f)=\left(\beta_{0}, \beta_{1}, \ldots, \beta_{n-1}\right)$ we obtain that $\beta_{i}=\left(c_{i}, c_{i+1}, \ldots, c_{i+b-1}\right) \in \mathbb{F}_{q}^{b}$, where

$$
c_{i+\ell}=\operatorname{Tr}\left(f\left(\eta^{i+\ell}\right)\right)
$$

for $0 \leq \ell \leq b-1$ and $0 \leq i \leq n-1$. It follows from Definition III.1 that $f^{(\ell)}(x)=f\left(\eta^{\ell} x\right)$ for $0 \leq \ell \leq b-1$. Hence we have that

$$
f^{(\ell)}\left(\eta^{i}\right)=f\left(\eta^{\ell} \eta^{i}\right)=f\left(\eta^{i+\ell}\right)
$$

for $0 \leq \ell \leq b-1$, and $0 \leq i \leq n-1$. Combining (4) and (5) we complete the proof.

Example 4: Let $q=4, n=21$ and $r=3$. Let $C$ be the cyclic code of length 21 over $\mathbb{F}_{4}$ considered in Examples 2 and 3. We keep the notation of Examples 2 and 3. In particular $\eta \in \mathbb{F}_{4^{3}}^{*}$ is a primitive 21-th root of 1 . Put $b=3$.

For an arbitrary element $\left(\beta_{0}, \beta_{1}, \ldots, \beta_{20}\right)$ of $C^{(3)} \in$ $\left(\mathbb{F}_{4} \times \mathbb{F}_{4} \times \mathbb{F}_{4}\right)^{21}$, there exist $a_{0}, a_{5}, a_{7}, a_{9}, a_{10} \in \mathbb{F}_{4^{3}}$ such that

$$
\begin{gathered}
\beta_{i}=\left(\operatorname{Tr}\left(a_{0}+a_{5} \eta^{5 i}+a_{7} \eta^{7 i}+a_{9} \eta^{9 i}+a_{10} \eta^{10 i}\right),\right. \\
\operatorname{Tr}\left(a_{0}+a_{5} \eta^{5+5 i}+a_{7} \eta^{7+7 i}+a_{9} \eta^{9+9 i}+a_{10} \eta^{10+10 i}\right) \\
\left.\operatorname{Tr}\left(a_{0}+a_{5} \eta^{10+5 i}+a_{7} \eta^{14+7 i}+a_{9} \eta^{18+9 i}+a_{10} \eta^{20+10 i}\right)\right)
\end{gathered}
$$

for $0 \leq i \leq 20$.

\section{IV. $b$-Symbol Weights For Some Cyclic Codes}

Throughout this section we assume that $C$ is a cyclic code of length $n$ dividing $q^{r}-1$ whose nonzero set is exactly one $q$-cyclotomic coset $U$ in $\mathbb{Z} / n \mathbb{Z}$. If $U=\{0\}$, then $C$ is a repetition code and any $b$-symbol Hamming weight of any nonzero codeword $c$ of $C$ is $n$ for any $1 \leq b \leq n-1$. Hence we further assume that there exists an integer $1 \leq u \leq n-1$ such that $u \in U$.

There is a close connection of the codes of this section to irreducible cyclic codes. We explain this connection explicitly after Theorem IV.3 below. It is well known that it is a notoriously difficult open problem to determine the weight distribution of irreducible cyclic codes in general (see, for example, [5]).

First we present a useful stability theorem. We start with some notation.

For $1 \leq t \leq n-1$, let $V(t)=\operatorname{Span}_{\mathbb{F}_{q}}\left\{1, \eta^{u}, \ldots, \eta^{(t-1) u}\right\}$. Note that on the difference of consecutive dimensions we have

$$
\left(\operatorname{dim}_{\mathbb{F}_{q}} V(t+1)-\operatorname{dim}_{\mathbb{F}_{q}} V(t)\right) \in\{0,1\} \text { for all } t .
$$

The following definition is useful.

Definition IV.1: Let $\mu$ be the largest positive integer $t$ such that $\operatorname{dim}_{\mathbb{F}_{q}} V(t)=t$.

The next lemma gives an alternative definition of $\mu$ and it shows that $\mu$ is independent from the choice of primitive $n$-th root of unity and from the choice of $u \in U$.

Lemma IV.2: Under notation above, for $\mu$ given in Definition IV.1 we have $\mu=\operatorname{dim}_{\mathbb{F}_{q}} \mathbb{F}_{q}\left(\eta^{u}\right)$, where $\mathbb{F}_{q}\left(\eta^{u}\right)$ is the smallest finite field extension of $\mathbb{F}_{q}$ containing $\eta^{u}$.

Proof: It follows from (6) and Definition IV.1 that $\mu$ is the smallest positive integer $t$ satisfying

$$
\eta^{u(t+i)} \in \operatorname{Span}_{\mathbb{F}_{q}}\left\{1, \eta^{u}, \ldots, \eta^{u(t-1)}\right\},
$$

for all integers $i \geq 0$. Equivalently $\mu$ is the smallest positive integer $t$ such that $\mathbb{F}_{q}\left[\eta^{u}\right] \in \operatorname{Span}_{\mathbb{F}_{q}}\left\{1, \eta^{u}, \ldots, \eta^{u(t-1)}\right\}$. This means that $\mathbb{F}_{q}\left(\eta^{u}\right)=\mathbb{F}_{q}\left[\eta^{u}\right]=\operatorname{Span}_{\mathbb{F}_{q}}\left\{1, \eta^{u}, \ldots\right.$, $\left.\eta^{u(t-1)}\right\}$.

Corollary 1: Under notation above, for $\mu$ given in Definition IV.1 the following equivalent characterizations hold:

- $\mu=\operatorname{dim}_{\mathbb{F}_{q}} \mathbb{F}_{q}\left(\eta^{u}\right)$.

- $\mu=\operatorname{dim}_{\mathbb{F}_{q}} C$.

- $\mu$ is the multiplicative order of $q$ modulo $\frac{n}{\operatorname{gcd}(n, u)}$.

- $\mu$ is the size of the $q$-cyclotomic coset $U$ (containing $u$ ) in $\mathbb{Z} / n \mathbb{Z}$.

Proof: The multiplicative order of $\eta^{u}$ is $\frac{n}{\operatorname{gcd}(n, u)}$. Hence we have that $\operatorname{dim}_{\mathbb{F}_{q}} \mathbb{F}_{q}\left(\eta^{u}\right)=\mu$ if and only if $\mu$ is the smallest integer $r$ such that $\frac{n}{\operatorname{gcd}(n, u)}$ divides $q^{r}-1$. In particular this means that $\mu$ is the multiplicative order of $q$ modulo $\frac{n}{\operatorname{gcd}(n, u)}$.

Let $U$ be the nonzero set of $C$. It follows from the definition of the nonzero set (see Subsection II-C) that $\operatorname{dim}_{\mathbb{F}_{q}} C$ is the size of $U$. Note that $U$ is the $q$-cyclotomic coset containing $u$ in $\mathbb{Z} / n \mathbb{Z}$ as the nonzero set of $C$ consists of exactly one $q$-cyclotomic coset by assumption in this section. The size of $U$ is the smallest integer $r$ such that $q^{r} u \equiv u \bmod n$. This means that the size of $U$ is the smallest integer $r$ such that $\frac{n}{\operatorname{gcd}(n, u)}$ divides $q^{r}-1$. Combining the arguments above we complete the proof. 
First we present our stability theorem in the special case of this section. Basically it says that the $b$-symbol Hamming weight enumerators of $C$ are the same for all $b$-symbol Hamming weights if $b \geq \operatorname{dim}_{\mathbb{F}_{q}}(C)$. There exists a nonempty stability region always except the trivial case that $\operatorname{dim}_{\mathbb{F}_{q}} C=$ $n-1$. We generalize the next result to arbitrary cyclic codes in Theorem V.2 below, whose proof is more involved.

Theorem IV.3: Assume that $\operatorname{gcd}(n, q)=1$. Let $C$ be a cyclic code of length $n$ such that its nonzero set is exactly one $q$-cyclotomic coset $U$ of $\mathbb{Z} / n \mathbb{Z}$. Assume that $U \neq\{0\}$ and let $u \in U$. Let $k=\operatorname{dim}_{\mathbb{F}_{q}} C$. For any integer $b$ in the interval $k \leq b \leq n-1$, the $b$-symbol Hamming weight enumerator of $C$ is the same as the $k$-symbol Hamming weight enumerator of $C$.

Proof: Let $f(x)=a x^{u} \in \mathbb{F}_{q^{r}}[x] \backslash\{0\}$ be an arbitrary nonzero polynomial in $P(\{u\})$. Let $c^{(k)}(f) \in C^{(k)}$ and $c^{(b)}(f) \in C^{(b)}$ be the corresponding codewords, where we refer to Theorem III.2 for the explicit descriptions of the codewords. Note that

$$
c^{(b)}(f)=\left(\underline{\operatorname{Tr}(f(\eta))}, \underline{\operatorname{Tr}\left(f^{(1)}(\eta)\right)}, \cdots, \underline{\operatorname{Tr}\left(f^{(b)}(\eta)\right)}\right) .
$$

Putting $c^{(b)}(f)=\left(c_{0}^{(b)}(f), c_{1}^{(b)}(f), \ldots, c_{n-1}^{(b)}(f)\right) \in\left(\mathbb{F}_{q}^{b}\right)^{n}$, for the symbols of $c^{(b)}(f)$ in the alphabet $\mathbb{F}_{q}^{b}$ we observe that

$$
c_{i}^{(b)}(f)=\left(\operatorname{Tr}\left(a \eta^{u i}\right), \operatorname{Tr}\left(\eta^{u} a \eta^{u i}\right), \cdots, \operatorname{Tr}\left(\eta^{(b-1) u} a \eta^{u i}\right)\right) .
$$

Similarly for the symbols of $c^{(k)}(f)$ in the alphabet $\mathbb{F}_{q}^{\mu}$ we observe that

$$
c_{i}^{(k)}(f)=\left(\operatorname{Tr}\left(a \eta^{u i}\right), \operatorname{Tr}\left(\eta^{u} a \eta^{u i}\right), \cdots, \operatorname{Tr}\left(\eta^{(k-1) u} a \eta^{u i}\right)\right) .
$$

Using Corollary 1 we get

$$
\begin{aligned}
Q & :=\operatorname{Span}_{\mathbb{F}_{q}}\left\{1, \eta^{u}, \ldots, \eta^{(k-1) u}\right\} \\
& =\operatorname{Span}_{\mathbb{F}_{q}}\left\{1, \eta^{u}, \ldots, \eta^{(b-1) u}\right\}
\end{aligned}
$$

Hence if $\alpha \in \mathbb{F}_{q^{r}}$, then

$$
\begin{aligned}
0= & \operatorname{Tr}(\alpha)=\operatorname{Tr}\left(\eta^{u} \alpha\right)=\cdots=\operatorname{Tr}\left(\eta^{(k-1) u} \alpha\right) \\
& \Longleftrightarrow 0=\operatorname{Tr}(\alpha)=\operatorname{Tr}\left(\eta^{u} \alpha\right)=\cdots=\operatorname{Tr}\left(\eta^{(b-1) u} \alpha\right) .
\end{aligned}
$$

Using (7) and (8) this implies that $c_{i}^{(k)}(f)$ contributes to the Hamming weight of the codeword $c^{(k)}(f)$ of length $n$ over the alphabet $\mathbb{F}_{q}^{k}$ if and only if $c_{i}^{(b)}(f)$ contributes to the Hamming weight of the codeword $c^{(b)}(f)$ of length $n$ over the alphabet $\mathbb{F}_{q}^{b}$. Therefore the values of the Hamming weights (defined over their respective alphabets) of $c^{(k)}(f)$ and $c^{(b)}(f)$ are the same. This completes the proof.

Remark 1: We note that Theorem IV.3 (and hence Theorem V.2 below) has useful engineering consequences in applications. For example it implies that increasing $b$ for $b$-symbol for error correcting does give any further advantage if $b \geq k$ for these codes.

Now we explain the connection of the codes of this section to irreducible cyclic codes. Let $m=\operatorname{gcd}(u, n)$ and put $\bar{n}=n / m$. Note that $\eta^{u i}=\eta^{u(i+\bar{n})}$ for $i \geq 0$ as $\eta^{u \bar{n}}=$ $\eta^{n \frac{u}{m}}=1$. For a codeword $c=\left(c_{0}, c_{1}, \ldots, c_{n-1}\right) \in C$ and a codeword $c^{(b)}=\left(c_{0}^{(b)}, c_{1}^{(b)}, \ldots, c_{n-1}^{(b)}\right) \in C^{(b)}$, let $\bar{c} \in \mathbb{F}_{q}^{\bar{n}}$ and $\bar{c}^{(b)} \in\left(\mathbb{F}_{q}\right)^{\bar{n}}$ be the corresponding elements defined as the shortenings

$$
\bar{c}=\left(c_{0}, c_{1}, \ldots, c_{\bar{n}-1}\right) \text { and } \bar{c}^{(b)}=\left(c_{0}^{(b)}, c_{1}^{(b)}, \ldots, c_{\bar{n}-1}^{(b)}\right)
$$

to the first $\bar{n}$ symbols. Let $\bar{C} \subseteq \mathbb{F}_{q}^{\bar{n}}$ and $\bar{C}^{(b)} \subseteq\left(\mathbb{F}_{q}^{b}\right)^{\bar{n}}$ be the codes defined as

$$
\bar{C}=\{\bar{c}: c \in C\} \text { and } \bar{C}^{(b)}=\left\{\bar{c}^{(b)}: c^{(b)} \in C^{(b)}\right\} .
$$

Using the fact that $\eta^{u i}=\eta^{u(i+\bar{n})}$ for $i \geq 0$ we observe $\pi_{b}(\bar{C})=\bar{C}^{(b)}$. Moreover, between the Hamming weights of $c, \bar{c}, c^{(b)}$ and $\bar{c}^{(b)}$ we have the relations

$$
w_{H}(\bar{c})=\frac{1}{m} w_{H}(c) \text { and } w_{H}\left(\bar{c}^{(b)}\right)=\frac{1}{m} w_{H}\left(c^{(b)}\right) .
$$

Let $\theta=\eta^{u} \in \mathbb{F}_{q^{r}}^{*}$, which is a primitive $\bar{n}$-th root of 1 . We observe that $\vec{C}$ is the irreducible cyclic code of length $\bar{n}$ over $\mathbb{F}_{q}$ having the trace representation

$$
\bar{C}=\left\{\left(\operatorname{Tr}\left(a \theta^{0}\right), \operatorname{Tr}\left(a \theta^{1}\right), \ldots, \operatorname{Tr}\left(a \theta^{\bar{n}-1}\right)\right): a \in \mathbb{F}_{q^{r}}\right\} .
$$

These arguments show that $C$ is obtained from $\bar{C}$ via $m$ times replication so that

$$
C=\{(\bar{c}, \bar{c}, \ldots, \bar{c}): \bar{c} \in \bar{C}\} .
$$

Next we study $b$-symbol Hamming weights of $C$ for $b \in\left\{1,2, \ldots, \operatorname{dim}_{\mathbb{F}_{q}} C\right\}$, which determine the whole $b$-symbol Hamming weights profile of all integers $1 \leq b \leq n-1$ as proved in Theorem IV.3. Recall that 1-symbol Hamming weight corresponds to the usual Hamming weight. First we consider the case of length $n=q^{r}-1$.

Theorem IV.4: Assume that $\operatorname{gcd}(n, q)=1$. Let $C$ be a cyclic code of length $n=q^{r}-1$ such that its nonzero set is exactly one $q$-cyclotomic coset $U$ of $\mathbb{Z} / n \mathbb{Z}$. Assume that $U \neq\{0\}$ and let $u \in U$. Let $k=\operatorname{dim}_{\mathbb{F}_{q}} C$. Put $N=\operatorname{gcd}\left(u, q^{r}-1\right)$ and $N_{1}=\operatorname{gcd}\left(\frac{q^{r}-1}{q-1}, N\right)$. Let $c \in C$ be an arbitrary nonzero codeword. For $1 \leq b \leq k$, let $w_{b}(c)$ denote the $b$-symbol Hamming weight of $c$. If $N_{1}=1$, then we have

$$
w_{b}(c)=\left(q^{b}-1\right) q^{r-b} .
$$

If $N_{1}>1$, then we have

$$
\begin{aligned}
& {\left[\frac{N\left(q^{b}-1\right)}{q^{b-1}}\left\lceil\frac{q^{r}-\left\lfloor\left(N_{1}-1\right) q^{r / 2}\right\rfloor}{q N}\right\rceil\right] } \\
\leq & w_{b}(c) \leq\left\lfloor\frac{N\left(q^{b}-1\right)}{q^{b-1}}\left\lfloor\frac{q^{r}+\left\lfloor\left(N_{1}-1\right) q^{r / 2}\right\rfloor}{q N}\right\rfloor\right\rfloor .
\end{aligned}
$$

Proof: Let $f(x)=a x^{u} \in \mathbb{F}_{q^{r}}[x] \backslash\{0\}$ be an arbitrary nonzero polynomial in $P(\{u\})$. Let $c^{(b)}(f) \in C^{(b)}$ be the corresponding codeword.

We use some methods of [12] and further techniques in this proof. We refer to Appendix A for notation and background on algebraic function fields. In Appendix A we provide necessary background on algebraic function fields in order to make the paper self-contained.

As $b \leq k$, it follows from Definition IV. 1 and Corollary 1 that $\operatorname{dim}_{\mathbb{F}_{q}} V(b)=b$. Let $W=\left\{\alpha \in \mathbb{F}_{q^{r}}: \operatorname{Tr}(\alpha)=\right.$ $\left.\operatorname{Tr}\left(\eta^{u} \alpha\right) \stackrel{q}{=} \cdots=\operatorname{Tr}\left(\eta^{(b-1) u} \alpha\right)=0\right\}$. As $\operatorname{dim}_{\mathbb{F}_{q}} V(b)=$ $b, W$ is an $\mathbb{F}_{q}$-linear subspace of codimension $b$ in $\mathbb{F}_{q^{r}}$. 
Let $A(T) \in \mathbb{F}_{q^{r}}[T]$ be the monic $q$-additive polynomial of degree $q^{b}$ which splits in $\mathbb{F}_{q^{r}}$ and which satisfies $W=$ $\left\{A(y): y \in \mathbb{F}_{q^{r}}\right\}$. For some properties, including existence and uniqueness of $A(T)$, we refer to [9] and [12, Section 3].

Let $F$ be the algebraic function field corresponding to the codeword $c^{(b)}(f)$ given by $F=\mathbb{F}_{q^{r}}(x, y)$ such that $A(y)=a x^{u}$. Let $V \subseteq \mathbb{F}_{q^{r}}$ be the subset consisting of the roots of $A(T)$. Note that $V$ is an $\mathbb{F}_{q}$-linear subspace of dimension $b$. Let $P \subseteq V \backslash\{0\}$ be a subset such that each one dimensional $\mathbb{F}_{q}$-linear subspace of $V$ contains exactly one nonzero element in $V$. Then $|P|=\left(q^{b}-1\right) /(q-1)$ and let $P=\left\{\theta_{1}, \ldots, \theta_{\left(q^{b}-1\right) /(q-1)}\right\}$ be an enumeration of $P$.

Let $j$ be an integer in the range $1 \leq j \leq\left(q^{b}-1\right) /(q-1)$. Let $c\left(\theta_{j} f\right) \in C$ be the codeword corresponding to $\theta_{j} a x^{u}$. Let $F_{j}$ be the algebraic function field corresponding to $c\left(\theta_{j} f\right)$ given by $F_{j}=\mathbb{F}_{q^{r}}\left(x, y_{j}\right)$ such that $y_{j}^{q}-y_{j}=\theta_{j} a x^{u}$. It is not difficult to observe that $F$ is the compositum of $F_{1}, F_{2}, \ldots, F_{\left(q^{b}-1\right) /(q-1)}$, that is to say the smallest extension field containing all $F_{1}, F_{2}, \ldots, F_{\left(q^{b}-1\right) /(q-1)}$.

There exists exactly one rational place of $F$ at infinity, which is the rational place of $F$ over the rational place of the rational function field $\mathbb{F}_{q^{r}}(x)$ corresponding to the pole of $(x)$. Let $N^{(\mathrm{aff})}(F)$ denote the number of affine rational places of $F$.

Consider the $i$-th symbol $c_{i}^{(b)}(f)=\left(\operatorname{Tr}\left(f\left(\eta^{i}\right)\right)\right.$, $\left.\operatorname{Tr}\left(\eta^{u} f\left(\eta^{i}\right)\right), \ldots, \operatorname{Tr}\left(\eta^{(b-1) u} f\left(\eta^{i}\right)\right) \in F_{q}^{b_{\ell}}\right)$ of the codeword $c^{(b)}(f)$ for $0 \leq i \leq n-1$. This symbol contributes to the Hamming weight $w_{H}\left(c^{(b)}(f)\right)$ of $c^{(b)}(f)$ if and only if there are $q^{b}$ distinct rational places of the covering $F / \mathbb{F}_{q^{r}}(x)$ over the place of the rational function field $\mathbb{F}_{q^{r}}(x)$ corresponding to the zero of $\left(x-\eta^{i}\right)$. Also there exist exactly $q^{b}$ distinct rational places of the covering $F / \mathbb{F}_{q^{r}}(x)$ over the place of the rational function field $\mathbb{F}_{q^{r}}(x)$ corresponding to the zero of $(x)$. Hence we get that

$$
\left(n-w_{H}\left(c^{(b)}(f)\right)\right) q^{b}+q^{b}=N^{(\text {aff })}(F) .
$$

This is equivalent to

$$
w_{H}\left(c^{(b)}(f)\right)=q^{r}-\frac{N^{(\mathrm{aff})}(F)}{q^{b}} .
$$

Recall that $j$ is an integer in the range $1 \leq j \leq\left(q^{b}-\right.$ 1)/ $(q-1)$. Again there exists exactly one rational place of $F_{j}$ at infinity. Let $N^{(\text {aff })}\left(F_{j}\right)$ denote the number of affine rational places of $F_{j}$. For the Hamming weight $w_{H}\left(c\left(\theta_{j} f\right)\right)$ of $c\left(\theta_{j} f\right)$ using similar arguments we also get that

$$
w_{H}\left(c\left(\theta_{j} f\right)\right)=q^{r}-\frac{N^{(\mathrm{aff})}\left(F_{j}\right)}{q} .
$$

Let $S$ and $S_{j}$ be the integers defined via

$$
N^{(\text {aff })}(F)=q^{r}-S \text { and } N^{(\text {aff })}\left(F_{j}\right)=q^{r}-S_{j} .
$$

It follows from [6, Corollary 6.7] (see also [10, Proposition 3.6] and [17, Lemma 2.4 and (3)]) that

$$
S=\sum_{j=1}^{\left(q^{r}-1\right) /(q-1)} S_{j} .
$$

Here we use the fact that $A(T)$ is a $q$-additive polynomial $\quad 510$ splitting in $\mathbb{F}_{q^{r}}$. Using (14), (15) and (16) yields

$$
\begin{aligned}
V:= & q^{r}-N^{(\mathrm{aff})}(F) \\
= & \sum_{j=1}^{\left(q^{r}-1\right) /(q-1)}\left(q^{r}-N^{(\mathrm{aff})}\left(F_{j}\right)\right) \\
= & \sum_{j=1}^{\left(q^{r}-1\right) /(q-1)}\left(-(q-1) q^{r}\right. \\
& \left.+q w_{H}\left(c\left(\theta_{j} f\right)\right)\right) \\
& -q^{r+b}+q^{r} \\
& +q \sum_{j=1}^{\left(q^{r}-1\right) /(q-1)} w_{H}\left(c\left(\theta_{j} f\right)\right) .
\end{aligned}
$$

This implies that

$$
w_{H}\left(c^{(b)}(f)\right)=\frac{1}{q^{b-1}} \sum_{j=1}^{\left(q^{b}-1\right) /(q-1)} w_{H}\left(c\left(\theta_{j} f\right)\right) .
$$

Recall that $N=\operatorname{gcd}\left(u, q^{r}-1\right)$. Put $\bar{n}=\frac{q^{r}-1}{N}$ and let $\bar{c}\left(\theta_{j} f\right)$ be the shortening of $c\left(\theta_{j} f\right)$ to the first $\bar{n}$ symbols as in (10). Similarly let $\bar{C}$ be the shortening of the code $C$ to the first $\bar{n}$ symbols as in (11). Note that $\bar{C}$ is an irreducible cyclic code of length $\bar{n}$ over $\mathbb{F}_{q}$ with $N=\frac{q^{r}-1}{\bar{n}}$.

Assume first that $N_{1}=1$. Using [5, Theorem 15] we have

$$
w_{H}\left(\bar{c}\left(\theta_{j} f\right)\right)=\frac{(q-1) q^{r-1}}{N}
$$

for each $1 \leq j \leq \frac{q^{b}-1}{q-1}$. Using (19) and (12) we obtain that

$$
w_{H}\left(c\left(\theta_{j} f\right)\right)=(q-1) q^{r-1}
$$

for each $1 \leq j \leq \frac{q^{b}-1}{q-1}$. Combining (20) and (18) we conclude that

$$
w_{b}(c(f))=w_{H}\left(c^{(b)}(f)\right)=\frac{q^{b}-1}{q^{b-1}} q^{r-1}=\left(q^{b}-1\right) q^{r-b}
$$

which completes the proof of the case that $N_{1}=1$.

Assume next that $N_{1}>1$. Using [5, Theorem 24] we have

$$
\begin{gathered}
(q-1)\left\lceil\frac{q^{r}-\left\lfloor\left(N_{1}-1\right) q^{r / 2}\right\rfloor}{q N}\right\rceil \leq w_{H}\left(\bar{c}\left(\theta_{j} f\right)\right) \\
\leq(q-1)\left\lfloor\frac{q^{r}+\left\lfloor\left(N_{1}-1\right) q^{r / 2}\right\rfloor}{q N}\right\rfloor
\end{gathered}
$$

for each $1 \leq j \leq \frac{q^{b}-1}{q-1}$. Using (21) and (12) we obtain that

$$
\begin{aligned}
& N(q-1)\left\lceil\frac{q^{r}-\left\lfloor\left(N_{1}-1\right) q^{r / 2}\right\rfloor}{q N}\right\rceil \leq w_{H}\left(c\left(\theta_{j} f\right)\right) \\
& \quad \leq N(q-1)\left\lfloor\frac{q^{r}+\left\lfloor\left(N_{1}-1\right) q^{r / 2}\right\rfloor}{q N}\right\rfloor
\end{aligned}
$$

for each $1 \leq j \leq \frac{q^{b}-1}{q-1}$. Combining (22) and (18) we conclude that

$$
\begin{aligned}
& N \frac{q^{b}-1}{q^{b-1}}\left\lceil\frac{q^{r}-\left\lfloor\left(N_{1}-1\right) q^{r / 2}\right\rfloor}{q N}\right\rceil \leq w_{b}(c(f)) \\
& \leq N \frac{q^{b}-1}{q^{b-1}}\left\lfloor\frac{q^{r}+\left\lfloor\left(N_{1}-1\right) q^{r / 2}\right\rfloor}{q N}\right\rfloor .
\end{aligned}
$$


As $w_{b}(C(f))$ is an integer, taking the ceiling and the floor integer parts of both sides of (23) we complete the proof.

Remark 2: Let $u^{*}$ be the largest positive divisor $t$ of $u$ such that $\operatorname{gcd}(t, q)=1$. The genus $g(F)$ of the function field $F$ in the proof of Theorem IV.4 is $g(F)=\frac{\left(q^{b}-1\right)\left(u^{*}-1\right)}{2}$. Hence Serre's improvement on the Hasse-Weil bound [16, Theorem 5.3.1] yields

$$
\left|N^{(\mathrm{aff})}(F)\right| \leq q^{r}+\frac{\left(q^{b}-1\right)\left(u^{*}-1\right)}{2}\left\lfloor 2 q^{r / 2}\right\rfloor .
$$

For a nonzero codeword $c \in C$, using the arguments in the proof of Theorem IV.4 we arrive at the bounds

$$
\begin{aligned}
& q^{r}-q^{r-b}-\left\lfloor\frac{\left(q^{b}-1\right)\left(u^{*}-1\right)\left\lfloor 2 q^{r / 2}\right\rfloor}{2 q^{b}}\right\rfloor \\
\leq & w_{b}(c) \\
\leq & q^{r}-q^{r-b}+\left\lfloor\frac{\left(q^{b}-1\right)\left(u^{*}-1\right)\left\lfloor 2 q^{r / 2}\right\rfloor}{2 q^{b}}\right\rfloor .
\end{aligned}
$$

The bounds of this remark are comparable to the bounds of Theorem IV.4. Nevertheless the bounds of Theorem IV.4 are better in general. We illustrate this in Example 5 below.

It is important to observe that the methods of this remark is valuable in the following sense. If the assumption of Theorem IV.4 that the nonzero set of $C$ is exactly one $q$-cyclotomic coset of $\mathbb{Z} / n \mathbb{Z}$ does not hold, then we cannot use [5] as in the proof of Theorem IV.4. This corresponds to the general situation of arbitrary cyclic codes. We consider arbitrary cyclic codes in Section V, where we develop and use the methods similar to the methods of this remark.

Example 5: We compare the bounds of Theorem IV.4 and Remark 2 in the following concrete cases.

- Case $q=3, b=2, r=10, u=11, n=q^{r}-1$.

Theorem IV.4: $50336 \leq w_{b}(c) \leq 54648$. Remark 2: $50328 \leq w_{b}(c) \leq 54648$.

- Case $q=3, b=2, r=10, u=61, n=q^{r}-1$.

Theorem IV.4: $39528 \leq w_{b}(c) \leq 65392$. Remark 2: $39528 \leq w_{b}(c) \leq 65448$.

- Case $q=2, b=2, r=10, u=11, n=q^{r}-1$. Theorem IV.4: $528 \leq w_{b}(c) \leq 1006$. Remark 2: $528 \leq w_{b}(c) \leq 1008$.

- Case $q=2, b=2, r=10, u=31, n=q^{r}-1$.

Theorem IV.4: $93 \leq w_{b}(c) \leq 1488$. Remark 2: $48 \leq w_{b}(c) \leq 1488$.

Using the methods in the proof of Theorem IV.4 and (12) we obtain our bounds for the general length $n \mid\left(q^{r}-1\right)$ in the next corollary.

Corollary 2: Assume that $\operatorname{gcd}(n, q)=1$. Let $C$ be a cyclic code of length $n \mid\left(q^{r}-1\right)$ such that its nonzero set is exactly one $q$-cyclotomic coset $U$ of $\mathbb{Z} / n \mathbb{Z}$. Assume that $U \neq\{0\}$ and let $u \in U$. Let $k=\operatorname{dim}_{\mathbb{F}_{q}} C$. Put $m=\operatorname{gcd}(u, n), N=\frac{q^{r}-1}{n} m$ and $N_{1}=\operatorname{gcd}\left(\frac{q^{r}-1}{q-1}, N\right)$. Let $c \in C$ be an arbitrary nonzero codeword. For $1 \leq b \leq k$, let $w_{b}(c)$ denote the $b$-symbol 585 Hamming weight of $c$. If $N_{1}=1$, then we have

$$
w_{b}(c)=\frac{m}{N}\left(q^{b}-1\right) q^{r-b} .
$$

If $N_{1}>1$, then we have

$$
\begin{aligned}
& {\left[\frac{m\left(q^{b}-1\right)}{q^{b-1}}\left\lceil\frac{q^{r}-\left\lfloor\left(N_{1}-1\right) q^{r / 2}\right\rfloor}{q N}\right\rceil\right] } \\
\leq & w_{b}(c) \leq\left\lfloor\frac{m\left(q^{b}-1\right)}{q^{b-1}}\left\lfloor\frac{q^{r}+\left\lfloor\left(N_{1}-1\right) q^{r / 2}\right\rfloor}{q N}\right\rfloor .\right.
\end{aligned}
$$

Remark 3: If $n=q^{r}-1$, then $m=N$ and Corollary 2 coincides with Theorem IV.4.

Remark 4: If $m=1$ and $b=1$, then Corollary 2 coincides with [5, Theorem 24].

Remark 5: Note that $k \leq r$ as the nonzero set of $C$ consists of only one $q$-cyclotomic coset of $\mathbb{Z} / n \mathbb{Z}$ in Corollary 2 . Moreover if $N_{1}=1$, then $N \mid(q-1)$. Hence the $b$-symbol Hamming weight $\frac{m}{N}\left(q^{b}-1\right) q^{r-b}$ in (24) is an integer.

If $N_{1}=1$, then using also Theorem IV.3 we determine the $b$-symbol Hamming weight enumerator of $C$ not only for $1 \leq k \leq b$ but for the full range $1 \leq b \leq n-1$ in this case.

Corollary 3: Keeping the notation and assumptions of Corollary 2, assume further that $N_{1}=1$. For integers $b$ in the interval $1 \leq b \leq n-1$, the $b$-symbol Hamming weight enumerator of $C$ is

$$
\begin{cases}1+\left(q^{k}-1\right) Z^{\frac{m\left(q^{b}-1\right) q^{r-b}}{N}} & \text { if } 1 \leq b \leq k \\ 1+\left(q^{k}-1\right) Z^{\frac{m\left(q^{k}-1\right)(q-k)}{N}} & \text { if } k+1 \leq b \leq n-1 .\end{cases}
$$

Proof: Assume first that $1 \leq b \leq k$, Then we have $w_{b}(c)=\frac{m}{N}\left(q^{b}-1\right) q^{r-b}$ using Corollary 2 for any nonzero codeword $c$ of $C$. For the zero codeword $c=0$ of $C$ it is clear that $w_{b}(c)=0$. These imply that the $b$-symbol Hamming weight enumerator of $C$ is

$$
1+\left(q^{k}-1\right) Z^{\frac{m\left(q^{b}-1\right) q^{r-b}}{N}} .
$$

In particular if $b=k$, then the $b$-symbol Hamming weight enumerator of $C$ is

$$
1+\left(q^{k}-1\right) Z^{\frac{m\left(q^{k}-1\right) q^{r-k}}{N}} .
$$

Using Theorem IV.3, the $b$-symbol Hamming weight enumerator of $C$ is exactly as in (25) if $k+1 \leq b \leq n-1$.

Further knowledge on the weight distribution of irreducible cyclic codes combined with the methods of the proof of Theorem IV.4 would immediately imply some improvements on the general bound of Corollary 2. Note that there exists such knowledge on the weight distribution on irreducible cyclic codes only for some very special subcases. We present a collection of such improvements on special subcases in the next corollary.

Corollary 4: Keeping the notation and assumptions of Corollary 2, we obtain improved bounds in the following special subcases. Let $q=p^{s}$, where $p$ is the characteristic of $\mathbb{F}_{q}$. Recall that $k=\operatorname{dim}_{\mathbb{F}_{q}} C$. 
- Assume further that $N_{1}=2$. We have:

$$
\begin{aligned}
& \left\lceil\frac{m\left(q^{b}-1\right)\left(q^{r}-q^{r / 2}\right)}{q^{b} N}\right\rceil \\
\leq & w_{b}(c) \\
\leq & \left\lfloor\frac{m\left(q^{b}-1\right)\left(q^{r}+q^{r / 2}\right)}{q^{b} N}\right\rfloor .
\end{aligned}
$$

- Assume further that $N_{1}=3, p \equiv 2 \bmod 3$ and $s k \equiv 0$ $\bmod 4$. We have:

$$
\begin{aligned}
& \left\lceil\frac{m\left(q^{b}-1\right)\left(q^{r}-q^{r / 2}\right)}{q^{b} N}\right\rceil \\
\leq & w_{b}(c) \\
\leq & \left\lfloor\frac{m\left(q^{b}-1\right)\left(q^{r}+2 q^{r / 2}\right)}{q^{b} N}\right\rfloor .
\end{aligned}
$$

- Assume further that $N_{1}=3, p \equiv 2 \bmod 3$ and $s k \equiv 2$ $\bmod 4$. We have:

$$
\begin{aligned}
& \left\lceil\frac{m\left(q^{b}-1\right)\left(q^{r}-2 q^{r / 2}\right)}{q^{b} N}\right\rceil \\
\leq & w_{b}(c) \\
\leq & \left\lfloor\frac{m\left(q^{b}-1\right)\left(q^{r}+q^{r / 2}\right)}{q^{b} N}\right\rfloor .
\end{aligned}
$$

- Assume further that $N_{1}=4$ and $p \equiv 3 \bmod 4$. We have:

$$
\begin{aligned}
& \left\lceil\frac{m\left(q^{b}-1\right)\left(q^{r}-q^{r / 2}\right)}{q^{b} N}\right\rceil \\
\leq & w_{b}(c) \\
\leq & \left\lfloor\frac{m\left(q^{b}-1\right)\left(q^{r}+3 q^{r / 2}\right)}{q^{b} N}\right\rfloor
\end{aligned}
$$

Proof: First we assume that $N_{1}=2$. We use the methods in the proof of Theorem IV.4 and we keep its notation. In particular $c^{(b)}$ and $\bar{c}^{(b)}$ denote the corresponding nonzero codewords as in the proof of Theorem IV.4. We have, as in (18), that

$$
w_{H}\left(c^{(b)}(f)\right)=\frac{1}{q^{b-1}} \sum_{j=1}^{\left(q^{b}-1\right) /(q-1)} w_{H}\left(c\left(\theta_{j} f\right)\right) .
$$

Here $\theta_{j}$ for $1 \leq j \leq\left(q^{b}-1\right) /(q-1)$ are chosen as in the proof of Theorem IV.4. Using (12) we also have

$$
w_{H}\left(\bar{c}\left(\theta_{j} f\right)\right)=\frac{1}{m} w_{H}\left(c\left(\theta_{j} f\right)\right)
$$

for $\left.1 \leq j \leq q^{b}-1\right) /(q-1)$. Using [5, Theorem 17] we further obtain

$$
\begin{gathered}
\frac{(q-1)\left(q^{r}-q^{r / 2}\right)}{q N} \leq w_{H}\left(\bar{c}\left(\theta_{j} f\right)\right) \\
\leq \frac{(q-1)\left(q^{r}+q^{r / 2}\right)}{q N}
\end{gathered}
$$

Combining (26), (27) and (28) we conclude that

$$
\begin{gathered}
\frac{m\left(q^{b}-1\right)\left(q^{r}-q^{r / 2}\right)}{q^{b} N} \leq w_{H}\left(\bar{c} c\left(\theta_{j} f\right)\right) \\
\leq \frac{\left(q^{b}-1\right)\left(q^{r}+q^{r / 2}\right)}{q^{b} N} .
\end{gathered}
$$

Taking the ceiling and floor integer parts of both sides of (29) we complete the proof of the case $N_{1}=2$.

Assume next that $N_{1}=3, p \equiv 2 \bmod 3$ and $s k \equiv 0$ mod 4. In this case, using [5, Theorem 19] we obtain

$$
\frac{(q-1)\left(q^{r}-q^{r / 2}\right)}{q N} \leq w_{H}\left(\bar{c}\left(\theta_{j} f\right)\right) \leq \frac{(q-1)\left(q^{r}+2 q^{r / 2}\right)}{q N}
$$

instead of (28) of the case $N_{1}=2$. Using the same arguments with this change we complete the proof of the current case.

Assume next that $N_{1}=3, p \equiv 2 \bmod 3$ and $s k \equiv 2$ mod 4. In this case, using [5, Theorem 19] we obtain

$$
\frac{(q-1)\left(q^{r}-2 q^{r / 2}\right)}{q N} \leq w_{H}\left(\bar{c}\left(\theta_{j} f\right)\right) \leq \frac{(q-1)\left(q^{r}+q^{r / 2}\right)}{q N}
$$

instead of (28) of the case $N_{1}=2$. Using the same arguments $\quad 680$ with this change we complete the proof of the current case.

Assume next that $N_{1}=3, p \equiv 2 \bmod 3$ and $s k \equiv 2$ mod 4. In this case, using [5, Theorem 20] we obtain

$$
\frac{(q-1)\left(q^{r}-q^{r / 2}\right)}{q N} \leq w_{H}\left(\bar{c}\left(\theta_{j} f\right)\right) \leq \frac{(q-1)\left(q^{r}+3 q^{r / 2}\right)}{q N}
$$

instead of (28) of the case $N_{1}=2$. Using the same arguments with this change we complete the proof of the current case. $\square$

Now we summarize and compare the bounds of this section. Theorem IV.4 is a special subcase of Corollary 2 with $n=$ $q^{r}-1$. In terms of the bounds, Corollary 3 is a special subcase of Corollary 2 with $N_{1}=1$. Corollary 4 improves Corollary 2 in some concrete cases only if $N_{1} \in\{2,3,4\}$. We present some concrete examples illustrating also these improvements below.

Example 6: We give concrete examples for the bounds of Corollary 3 and Corollary 4.

- Case $q=2, b=2, r=12, u=11, n=1365$.

Corollary 2: $993 \leq w_{b}(c) \leq 1056$. Corollary 4: $1008 \leq w_{b}(c) \leq 1056$.

- Case $q=2, b=2, r=10, u=5, n=341$.

Corollary 2: $240 \leq w_{b}(c) \leq 271$. Corollary 4: $240 \leq w_{b}(c) \leq 264$.

- Case $q=3, b=2, r=8, u=7, n=1640$.

$$
\text { Corollary 2: } 1406 \leq w_{b}(c) \leq 1512 .
$$$$
\text { Corollary 4: } 1440 \leq w_{b}(c) \leq 1512 .
$$

- Case $q=9, b=3, r=8, u=47, n=10761680$. Corollary 2: $10742009 \leq w_{b}(c) \leq 10751832$. Corollary 4: $10745280 \leq w_{b}(c) \leq 10751832$.

- Case $q=2, b=2, r=16, u=17, n=3855$.

$$
\text { Corollary 2: } 2712 \leq w_{b}(c) \leq 3072 .
$$

Corollary 4 does not work in this case as $N_{1}=17$ in this case. 
Remark 6: As we consider cyclic and hence linear codes throughout this paper, our lower and upper bounds on the $b$-symbol Hamming weights of nonzero codewords mean lower and upper bounds on the $b$-symbol Hamming distances between distinct codewords. Hence our bounds throughout this paper also correspond to lower and upper bounds on $b$-symbol Hamming distance of the codes we consider.

\section{V. $b$-Symbol Weights For ARbitrary Cyclic Codes}

Throughout this section we assume that $C$ is a cyclic code of length $n$ dividing $q^{r}-1$. Let $U$ be the nonzero set of $C$ and let $U_{1}, \ldots, U_{\rho}$ be the distinct $q$-cyclotomic cosets of $\mathbb{Z} / n Z$ included in $U$. Note that $U=U_{1} \sqcup U_{2} \cdots \sqcup U_{\rho}$ and $\rho \geq 1$, where $\sqcup$ indicates that the sets $U_{1}, \ldots, U_{\rho}$ in the union are pairwise disjoint. As in Section IV we assume that $U \neq\{0\}$ in order to avoid the trivial case. Choose $u_{j} \in U_{j}$ and put $k_{j}=\left|U_{j}\right|$ for $1 \leq j \leq \rho$. Note that for the $\mathbb{F}_{q}$-dimension $k$ of $C$ we have $k=k_{1}+\cdots+k_{\rho}$.

We first generalize our stability theorem (see Theorem IV.3) to arbitrary cyclic codes. Recall that $\eta \in \mathbb{F}_{q^{r}}^{*}$ is a primitive $n$-th root of 1 . We introduce some notation. For $0 \leq t \leq n-1$, let $\mathbf{v}_{t}$ be the vector in $\mathbb{F}_{q^{r}}^{\rho}$ defined as

$$
\mathbf{v}_{t}=\left[\eta^{t u_{1}}, \eta^{t u_{2}}, \ldots, \eta^{t u_{\rho}}\right] .
$$

For $1 \leq t \leq n-1$, let $V(t) \subseteq \mathbb{F}_{q^{r}}^{\rho}$ be the $\mathbb{F}_{q^{-}}$-linear subspace defined as

$$
V(t)=\operatorname{Span}_{\mathbb{F}_{q}}\left\{\mathbf{v}_{0}, \mathbf{v}_{1}, \ldots, \mathbf{v}_{t-1}\right\} .
$$

The following lemma is useful.

Lemma V.1: Under the above notation, we have

$$
\operatorname{dim}_{\mathbb{F}_{q}} V(t)=\left\{\begin{array}{cl}
t & \text { if } 1 \leq t \leq k-1 \\
k & \text { if } k \leq t \leq n-1
\end{array}\right.
$$

Moreover, $\left\{\mathbf{v}_{0}, \ldots, \mathbf{v}_{t-1}\right\}$ is an $\mathbb{F}_{q}$-basis of $V(t)$ if $1 \leq t \leq$ $k-1$. Also $\left\{\mathbf{v}_{0}, \ldots, \mathbf{v}_{k-1}\right\}$ is an $\mathbb{F}_{q}$-basis of $V(t)$ if $k \leq$ $t \leq n-1$.

Proof: Recall that $\mathbb{F}_{q}\left(\eta^{u_{j}}\right)$ denotes the smallest finite field extension over $\mathbb{F}_{q}$ containing $\eta^{u_{j}}$. For the index of this extension we have $\left[\mathbb{F}_{q}\left(\eta^{u_{j}}\right): \mathbb{F}_{q}\right]=k_{j}$. Let $m_{j}(x) \in \mathbb{F}_{q}[x]$ be the minimal polynomial of $\eta^{j}$ over $\mathbb{F}_{q}$. It is clear that $\operatorname{deg} m_{j}(x)=k_{j}$ and the set $\left\{m_{1}(x), m_{2}(x), \ldots, m_{\rho}(x)\right\}$ consists of irreducible polynomials over $\mathbb{F}_{q}$ and the elements of this set are pairwise distinct.

We first show that $\operatorname{dim}_{\mathbb{F}_{q}} V(k)=k$. Assume the contrary, and let $e_{0}, e_{1}, \ldots, e_{k-1} \in \mathbb{F}_{q}$ such that

$$
e_{0} \mathbf{v}_{0}+e_{1} \mathbf{v}_{1}+\cdots+e_{k-1} \mathbf{v}_{k-1}=\mathbf{0} .
$$

Let $1 \leq j \leq \rho$. Considering the $j$-th coordinates of the both sides of (31) we get

$$
e_{0}+e_{1} \eta^{u_{j}}+\cdots+e_{k-1} \eta^{(k-1) u_{j}}=0 .
$$

Let $h(x)=e_{0}+e_{1} x+\cdots+e_{k-1} x^{k-1} \in \mathbb{F}_{q}[x]$, which is a nonzero polynomial of degree at most $k-1$. It follows from (32) that $\eta^{u_{j}}$ is a root of $h(x)$. Hence we conclude that

$$
h\left(\eta^{u_{j}}\right)=0 \text { for each } 1 \leq j \leq \rho .
$$

As $m_{j}(x)$ is the minimal polynomial of $\eta^{u_{j}}$ over $\mathbb{F}_{q}$ and $h(x) \in \mathbb{F}_{q}[x]$ we obtain that

$$
m_{j}(x) \mid h(x) \text { for each } 1 \leq j \leq \rho .
$$

Recall that $\left\{m_{1}(x), m_{2}(x), \ldots, m_{\rho}(x)\right\}$ consists of irreducible polynomials over $\mathbb{F}_{q}$ and that the elements of this set are pairwise distinct. These arguments yield that the polynomial $\prod_{j=1}^{\rho} m_{j}(x)$ divides $h(x)$ and hence

$$
\operatorname{deg} h(x) \geq \sum_{i=1}^{\rho} \operatorname{deg} m_{j}(x)=\sum_{j=1}^{\rho} k_{j}=k .
$$

This is a contradiction as $h(x)$ is a nonzero polynomial of degree at most $k-1$.

It is clear that $V(t-1) \subseteq V(t)$ and

$$
0 \leq \operatorname{dim}_{\mathbb{F}_{q}} V(t)-\operatorname{dim}_{\mathbb{F}_{q}} V(t-1) \leq 1
$$

for each $2 \leq t \leq n-1$. Moreover, $V(1)=\operatorname{Span}_{\mathbb{F}_{q}}\{[1, \ldots, 1]\}$ and hence $\operatorname{dim}_{\mathbb{F}_{q}} V(1)=1$. Combining (33), and the facts $\operatorname{dim}_{\mathbb{F}_{q}} V(1)=1, \operatorname{dim}_{\mathbb{F}_{q}} V(k)=k$ we conclude that $\operatorname{dim}_{\mathbb{F}_{q}} V(t)=t$ for each integer $t$ in the range $1 \leq t \leq k$. Moreover, these also imply that

$$
\left\{\mathbf{v}_{0}, \mathbf{v}_{1}, \ldots, \mathbf{v}_{t}\right\}
$$

is a basis of $V(t)$ for each integer $t$ in the range $1 \leq t \leq k$.

It remains to prove that $V(k+i) \subseteq V(k)$ for $1 \leq i \leq$ $n-k-1$. We prove this by induction on $i$. First we consider the induction step $i=1$. Let $m(x)=m_{1}(x) m_{2}(x) \cdots m_{\rho}(x)$, which is a monic polynomial of degree $k$. Considering the coefficients of $m(x)$ let

$$
m(x)=x^{k}+e_{k-1} x^{k-1}+\cdots+e_{1} x+e_{0},
$$

where $e_{k-1}, \ldots, e_{1}, e_{0} \in \mathbb{F}_{q}$. As $m\left(\eta^{u_{j}}\right)=0$ for each $1 \leq$ $j \leq \rho$, the arguments above in this proof imply that

$$
\mathbf{v}_{k}+e_{k-1} \mathbf{v}_{k-1}+\cdots+e_{1} \mathbf{v}_{1}+e_{0} \mathbf{v}_{0}=\mathbf{0}
$$

This shows that $\mathbf{v}_{k} \in V(k)$ and hence $V(k+1) \subseteq V(k)$. Assume the induction hypothesis that $V(k+i) \subseteq V(k)$. Let $h(x)=x^{i} m(x)$, which is a monic polynomial of degree $k+i$. Considering the coefficients of $h(x)$ let

$$
k(x)=x^{k+i}+e_{k+i-1} x^{k+i-1}+\cdots+e_{1} x+e_{0},
$$

where $e_{k+i-1}, \ldots, e_{1}, e_{0} \in \mathbb{F}_{q}$. Similarly we obtain that

$$
\mathbf{v}_{k+i}+e_{k_{i}-1} \mathbf{v}_{k_{i}-1}+\cdots+e_{1} \mathbf{v}_{1}+e_{0} \mathbf{v}_{0}=\mathbf{0} .
$$

This yields $\mathbf{v}_{k+i} \in V(k+i)$ and hence $V(k+i+1) \subseteq V(k+i)$. This completes the proof.

Next we present our stability theorem for arbitrary cyclic codes. Again it says, but now for arbitrary cyclic codes, that the $b$-symbol Hamming weight enumerators of $C$ are the same (and hence stable) for all $b$-symbol Hamming weights if $b \geq \operatorname{dim}_{\mathbb{F}_{q}}(C)$ (see also Theorem IV.3). There exists a nonempty stability region for $b$ except the trivial case that $\operatorname{dim}_{\mathbb{F}_{q}} C=n-1$.

Theorem V.2: Assume that $\operatorname{gcd}(n, q)=1$. Let $C$ be an arbitrary cyclic code of length $n$ and $U$ be its nonzero set in $\mathbb{Z} / n \mathbb{Z}$. Assume that $U \neq\{0\}$. Let $k=\operatorname{dim}_{\mathbb{F}_{q}} C$. For any 
integer $b$ in the interval $k \leq b \leq n-1$, the $b$-symbol Hamming weight enumerator of $C$ is the same as the $k$-symbol Hamming weight enumerator of $C$.

Proof: We use the notation fixed in this section so that $\left\{u_{1}, u_{2}, \ldots, u_{\rho}\right\}$ is a basic nonzero set of $C$. Let $f(x)=$ $a_{1} x^{u_{1}}+a_{2} x^{u_{2}}+\cdots+a_{\rho} x^{u_{\rho}} \in \mathbb{F}_{q^{r}}[x] \backslash\{0\}$ be an arbitrary nonzero polynomial in $P\left(\left\{u_{1}, u_{2}, \ldots, u_{\rho}\right\}\right)$. Let $c^{(k)}(f) \in$ $C^{(k)}$ and $c^{(b)}(f) \in C^{(b)}$ be the corresponding codewords. Note that

$$
c^{(b)}(f)=\left(\underline{\operatorname{Tr}(f(\eta))}, \underline{\operatorname{Tr}\left(f^{(1)}(\eta)\right)}, \cdots, \underline{\operatorname{Tr}\left(f^{(b)}(\eta)\right)}\right),
$$

where $f^{(t)}(x)$ is defined in Definition III.1. Namely we have

$$
f^{(t)}(x)=\eta^{t u_{1}} a_{1} x^{u_{1}}+\eta^{t u_{2}} a_{2} x^{u_{2}}+\cdots+\eta^{t u_{\rho}} a_{\rho} x^{u_{\rho}} .
$$

Let $i$ be an integer in the range $0 \leq i \leq n-1$. Let $c_{i}^{(b)}(f) \in \mathbb{F}_{q}^{b}$ be the $i$-th symbol of the codeword $c^{(b)}(f) \in\left(\mathbb{F}_{q}^{b}\right)^{n}$ so that

$$
c^{(b)}(f)=\left(c_{0}^{(b)}(f), c_{1}^{(b)}(f), \ldots, c_{n-1}^{(b)}(f)\right) .
$$

Let $y_{1}=a_{1} \eta^{i u_{1}}, y_{2}=a_{2} \eta^{i u_{2}}, \ldots, y_{\rho}=a_{\rho} \eta^{i u_{\rho}}$ all in $\mathbb{F}_{q^{r}}^{*}$. Note that

$$
\begin{aligned}
c_{i}^{(b)}(f)= & \left(\operatorname{Tr}\left(y_{1}+y_{2}+\cdots+y_{\rho}\right),\right. \\
& +\operatorname{Tr}\left(\eta^{u_{1}} y_{1}+\eta^{u_{2}} y_{2}+\cdots+\eta^{u_{\rho}} y_{\rho}\right), \cdots, \\
& +\operatorname{Tr}\left(\eta^{(b-1) u_{1}} y_{1}+\eta^{(b-1) u_{2}} y_{2}+\cdots\right. \\
& \left.\left.+\eta^{(b-1) u_{\rho}} y_{\rho}\right)\right) .
\end{aligned}
$$

Similarly for the $i$-th symbol $c_{i}^{(k)}(f) \in \mathbb{F}_{q}^{k}$ of the codeword $c^{(k)}(f) \in\left(\mathbb{F}_{q}^{k}\right)^{n}$ we have

$$
\begin{aligned}
c_{i}^{(k)}(f)= & \left(\operatorname{Tr}\left(y_{1}+y_{2}+\cdots+y_{\rho}\right),\right. \\
& +\operatorname{Tr}\left(\eta^{u_{1}} y_{1}+\eta^{u_{2}} y_{2}+\cdots+\eta^{u_{\rho}} y_{\rho}\right), \cdots, \\
& +\operatorname{Tr}\left(\eta^{(k-1) u_{1}} y_{1}+\eta^{(k-1) u_{2}} y_{2}+\cdots\right. \\
& \left.\left.+\eta^{(k-1) u_{\rho}} y_{\rho}\right)\right)
\end{aligned}
$$

Hence $c_{i}^{(b)}(f)$ does not contribute to the Hamming weight of the codeword $c^{(b)}(f)$ if and only if

$$
\begin{aligned}
0 & =\operatorname{Tr}\left(y_{1}+y_{2}+\cdots+y_{\rho}\right) \\
& =\operatorname{Tr}\left(\eta^{u_{1}} y_{1}+\eta^{u_{2}} y_{2}+\cdots+\eta^{u_{\rho}} y_{\rho}\right) \\
& \vdots \\
& =\operatorname{Tr}\left(\eta^{(b-1) u_{1}} y_{1}+\eta^{(b-1) u_{2}} y_{2}+\cdots+\eta^{(b-1) u_{\rho}} y_{\rho}\right) .
\end{aligned}
$$

Similarly $c_{i}^{(k)}(f)$ does not contribute to the Hamming weight of the codeword $c^{(k)}(f)$ if and only if

$$
\begin{aligned}
0 & =\operatorname{Tr}\left(y_{1}+y_{2}+\cdots+y_{\rho}\right) \\
& =\operatorname{Tr}\left(\eta^{u_{1}} y_{1}+\eta^{u_{2}} y_{2}+\cdots+\eta^{u_{\rho}} y_{\rho}\right) \\
& \vdots \\
& =\operatorname{Tr}\left(\eta^{(k-1) u_{1}} y_{1}+\eta^{(k-1) u_{2}} y_{2}+\cdots+\eta^{(k-1) u_{\rho}} y_{\rho}\right) .
\end{aligned}
$$

We will prove the following claim at the end of this proof.

Claim 1. The conditions in (35) and (36) are equivalent.
Assume Claim 1 holds. The weight of the contribution of the symbol $c_{i}^{(b)}(f)$ to the codeword $c^{(b)}(f)$ is 0 or 1 , which is identified with the condition in (35). The same holds for the symbol $c_{i}^{(k)}(f)$ to the codeword $c^{(k)}(f)$ and the condition (36). Using Claim 1 and running through all indices $0 \leq i \leq n-1$ we complete the proof.

Now we prove Claim 1 . As $k \leq b$ it is clear that (35) implies (36). Conversely assume that (36) holds. Let $t$ be an integer in the range $k \leq t \leq b-1$. Note that

$$
\left[\eta^{t u_{1}}, \eta^{t u_{2}}, \ldots, \eta^{t u_{\gamma}}\right]=\mathbf{v}_{t},
$$

where $\mathbf{v}_{t}$ is defined in (30). Using Lemma V.1 we obtain that $\mathbf{v}_{t} \in V(k)$ and hence there exist $e_{0}, e_{1}, \ldots, e_{k-1} \in \mathbb{F}_{q}$ such that

$$
\begin{aligned}
U:= & {\left[\eta^{t u_{1}}, \eta^{t u_{2}}, \ldots, \eta^{t u_{\gamma}}\right] } \\
= & e_{0}[1,1, \ldots, 1] \\
& +e_{1}\left[\eta^{u_{1}}, \eta^{u_{2}}, \ldots, \eta^{u_{\gamma}}\right] \\
& +\cdots \\
& +e_{k-1}\left[\eta^{(k-1) u_{1}}, \eta^{(k-1) u_{2}}, \ldots, \eta^{(k-1) u_{\gamma}}\right] .
\end{aligned}
$$

Multiplying both sides with $\left[y_{1}, \ldots, y_{\rho}\right]$ using the Euclidean 865 inner product in $\mathbb{F}_{q^{r}}^{\rho}$ we get

$$
\begin{aligned}
A:= & \eta^{t u_{1}} y_{1}+\eta^{t u_{2}} y_{2}+\cdots+\eta^{t u_{\gamma}} y_{\rho} \\
= & e_{0}\left(y_{1}+y_{2}+\cdots+y_{\rho}\right) \\
& +e_{1}\left(\eta^{u_{1}} y_{1}+\eta^{u_{2}} y_{2}+\cdots+\eta^{u_{\rho}} y_{\rho}\right)+\cdots \\
& +e_{k-1}\left(\eta^{(k-1) u_{1}} y_{1}+\eta^{(k-1) u_{2}} y_{2}+\cdots+\eta^{(k-1) u_{\rho}} y_{\rho}\right) .
\end{aligned}
$$

Taking trace of both sides and noting $e_{0}, e_{1}, \ldots, e_{k-1} \in \mathbb{F}_{q}$ yield

$$
\begin{aligned}
& \operatorname{Tr}\left(\eta^{t u_{1}} y_{1}+\eta^{t u_{2}} y_{2}+\cdots+\eta^{t u_{\gamma}} y_{\rho}\right) \\
& =e_{0} \operatorname{Tr}\left(y_{1}+y_{2}+\cdots+y_{\rho}\right) \\
& \quad+e_{1} \operatorname{Tr}\left(\eta^{u_{1}} y_{1}+\eta^{u_{2}} y_{2}+\cdots+\eta^{u_{\rho}} y_{\rho}\right) \\
& \quad \vdots \\
& \quad+e_{k-1} \operatorname{Tr}\left(\eta^{(k-1) u_{1}} y_{1}+\cdots+\eta^{(k-1) u_{\rho}} y_{\rho}\right) .
\end{aligned}
$$

As (36) holds by assumption, we have

$$
\begin{aligned}
0 & =\operatorname{Tr}\left(y_{1}+y_{2}+\cdots+y_{\rho}\right) \\
& =\operatorname{Tr}\left(\eta^{u_{1}} y_{1}+\eta^{u_{2}} y_{2}+\cdots+\eta^{u_{\rho}} y_{\rho}\right) \\
& \vdots \\
& =\operatorname{Tr}\left(\eta^{(k-1) u_{1}} y_{1}+\cdots+\eta^{(k-1) u_{\rho}} y_{\rho}\right) .
\end{aligned}
$$

for these terms in the right hand side of (37). Therefore using (37) we conclude that

$$
\operatorname{Tr}\left(\eta^{t u_{1}} y_{1}+\eta^{t u_{2}} y_{2}+\cdots+\eta^{t u_{\gamma}} y_{\rho}\right)=0 .
$$

This conclusion holds for each integer $t$ in the range $k \leq t \leq$ $b-1$, which completes the proof of Claim 1 .

Theorem V.2 implies that it is enough to study $b$-symbol Hamming weights of an arbitrary cyclic code $C$ of dimension $k$ only for $1 \leq b \leq k$ instead of the much larger integral interval $1 \leq b \leq n-1$ in general.

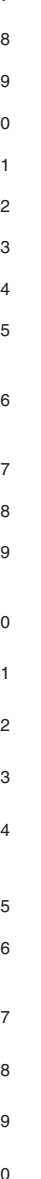$$
\text { . }
$$

2


Next we present our bounds on $b$-symbol Hamming weights on arbitrary cyclic codes for $1 \leq b \leq k$. We will need the following condition if $q$ is not a prime.

Condition V.3: Assume that $\operatorname{gcd}(n, q)=1$ and let $1 \leq u \leq$ $n-1$. Let $\bar{u}$ be the $q$-cyclotomic coset of $\mathbb{Z} / n \mathbb{Z}$ containing $u$, namely $\bar{u}=\left\{u q^{i} \bmod n: 0 \leq i \leq n-1\right\}$. Let $S(u)$ be the subset of $\bar{u}$ given by $S(u)=\{v \in \bar{u}: \operatorname{gcd}(v, q)=1\}$. If $u \neq 0$, then we say that $u$ satisfies Condition V.3 if both of the followings are satisfied:

- $S(u)$ is not empty.

- $u=\min S(u)$.

If $u=0$, then we say that $u$ satisfies Condition V.3.

Remark 7: If $q$ is a prime, then $u$ satisfies Condition V.3 if $u$ is the smallest element in $\bar{u}$. Hence if $q$ is a prime then Condition V.3 is satisfied automatically as we are free to choose any element from $\bar{u}$ in considering $C$.

Remark 8: If $q$ is not a prime, then there may be some $q$-cyclotomic cosets which do not satisfy Condition V.3. However, there is a rich collection of nontrivial $C$ such that Condition V.3 is satisfied and $q$ is not prime so that we present our results for arbitrary $q$. Now we give some toy examples in order to illustrate why Condition V.3 is needed in some cases. Let $q=4, r=2, n=q^{r}-1$. The $q$-cyclotomic cosets $\{10\}$ and $\{2,8\}$ have no element $u$ such that $u$ satisfies Condition V.3. For the $q$-cyclotomic coset $Z_{1}=\{1,4\}$, the element $u=1$ satisfies Condition V.3 and it is the smallest element of $Z_{1}$ as in the case that $q$ is a prime. However, for the $q$-cyclotomic coset $Z_{2}=\{6,9\}$, the element $u=9$ satisfies Condition V.3 but 9 is not the smallest element of $Z_{2}$. This is different from the case that $q$ is a prime (see Remark 7).

Remark 9: In our proofs in the rest of this section we apply Hasse Weil bound to Artin-Schreier type curves

$$
A(y)=a_{1} x^{u_{1}}+a_{2} x^{u_{2}}+\cdots+a_{\rho} x^{u_{\rho}},
$$

over $\mathbb{F}_{q^{r}}$, where $A(y)$ are certain additive polynomials. Condition V.3 guarantees that the curve in (38) is absolutely irreducible over $\mathbb{F}_{q^{r}}$. This is automatically satisfied by choosing the smallest choice of $u_{i}$ in each $q$-cyclotomic coset of $C$ if $q$ is a prime. If $q$ is not a prime and Condition V.3 is not satisfied, then we need to consider further methods. For example, if the curve in (38) has irreducible components, then applying Hasse-Weil bound to all of the irreducible components gives similar bounds on the weight of the cyclic code. However, this would be very complicated depending on $\left\{u_{1}, u_{2}, \ldots, u_{\rho}\right\}$ as we need to consider all $\left(a_{1}, a_{2}, \ldots, a_{\rho}\right) \in \mathbb{F}_{q^{r}}^{\rho} \backslash\{(0,0, \ldots, 0)\}$. There is a general method presented in [11] that uses involved symbolic computations and tools from algebra for studying all possible irreducible components in order to get such bounds on the weight of the cyclic code. If $\rho=1$, then all these are simple and implicitly used in Remark 2.

We first consider the case of length $n=q^{r}-1$ as we use methods from algebraic function fields (see also [18]). We extend our results to arbitrary length $n \mid\left(q^{r}-1\right)$ in Remark 14 below.

In the next theorem we present our bound in the case $b \leq \min \left\{k_{1}, k_{2}, \ldots, k_{\rho}\right\}$. Note that this case is much more general than the case of Section IV. Indeed it is possible, for example, that $k_{1}=k_{2}=\ldots=k_{\rho}$ and $\rho$ is a large positive integer.

Theorem V.4: Let $C$ be an arbitrary cyclic code of length $n=q^{r}-1$ over $\mathbb{F}_{q}$. Let $U_{0}=\left\{u_{1}, u_{2}, \ldots, u_{\rho}\right\}$ be a basic nonzero set of $C$. Assume that $U_{0} \neq\{0\}$ and each element of $U_{0}$ satisfies Condition V.3. Put $u^{*}=\max \left\{u_{1}, u_{2}, \ldots, u_{\rho}\right\}$. Let $\eta \in \mathbb{F}_{q^{r}}^{*}$ be a primitive $n$-th root of 1 . For $1 \leq j \leq \rho$, let $k_{j}$ be the index $\left[\mathbb{F}_{q}\left(\eta^{u_{j}}\right): \mathbb{F}_{q}\right]$ of the field extension $\mathbb{F}_{q}\left(\eta^{u_{j}}\right) / \mathbb{F}_{q}$. Let $c \in C$ be an arbitrary nonzero codeword. For $1 \leq b \leq$ $\min \left\{k_{1}, k_{2}, \ldots, k_{\rho}\right\}$, let $w_{b}(c)$ denote the $b$-symbol Hamming weight of $c$. We have

$$
\begin{aligned}
& q^{r}-q^{r-b}-\left\lfloor\frac{\left(q^{b}-1\right)\left(u^{*}-1\right)\left\lfloor 2 q^{r / 2}\right\rfloor}{2 q^{b}}\right\rfloor \\
\leq & w_{b}(c) \\
\leq & q^{r}-q^{r-b}+\left\lfloor\frac{\left(q^{b}-1\right)\left(u^{*}-1\right)\left\lfloor 2 q^{r / 2}\right\rfloor}{2 q^{b}}\right\rfloor .
\end{aligned}
$$

Proof: Let $f(x)=a_{1} x^{u_{1}}+a_{2} x^{u_{2}}+\cdots+$ $a_{\rho} x^{u_{\rho}} \in \mathbb{F}_{q^{r}}[x] \backslash\{0\}$ be an arbitrary nonzero polynomial in $P\left(\left\{u_{1}, \ldots, u_{\rho}\right\}\right)$. Let $c^{(b)}(f) \in C^{(b)}$ be the corresponding codeword. Recall that

$$
f^{(t)}(x)=\eta^{t u_{1}} a_{1} x^{u_{1}}+\eta^{t u_{2}} a_{2} x^{u_{2}}+\cdots+\eta^{t u_{\rho}} a_{\rho} x^{u_{\rho}}
$$

for $0 \leq t \leq b-1$, where $f^{(0)}(x)=f(x)$. Let $L \subseteq \mathbb{F}_{q^{r}}[x]$ be the $\mathbb{F}_{q}$-linear subspace of polynomials defined as

$$
L=\operatorname{Span}_{\mathbb{F}_{q}}\left\{f(x), f^{(1)}(x), \ldots, f^{(b-1)}(x)\right\} .
$$

First we show that $\operatorname{dim}_{\mathbb{F}_{q}} L=b$. Indeed assume the contrary that there exists $\left(e_{0}, e_{1}, \ldots, e_{b-1}\right) \in \mathbb{F}_{q}^{n} \backslash\{[0,0, \ldots, 0]\}$ such that

$$
e_{0} f(x)+e_{1} f^{(1)}(x)+\cdots+e_{b-1} f^{(b-1)}(x)=0 .
$$

Note that the polynomial in the left hand side of (40) has monomials with possibly nonzero coefficients only at $x^{u_{1}}, x^{u_{2}}, \ldots, x^{u_{\rho}}$. As $f(x) \neq 0$, there exists at least one coefficient $a_{j_{0}}$ such that $a_{j_{0}} \neq 0$. Using (39), (40) and the coefficient of the monomial $x^{u_{j}}$ in the left hand side of (40) we obtain that

$$
e_{0}+e_{1} \eta^{u_{j_{0}}}+e_{2} \eta^{2} u_{j_{0}}+\cdots+\eta^{(b-1) u_{j_{0}}}=0 .
$$

Let $e(x) \in \mathbb{F}_{q}[x]$ be the nonzero polynomial of degree at most $b-1$ such that

$$
e(x)=e_{0}+e_{1} x+\cdots+e_{b-1} x^{b-1} .
$$

Let $m_{j_{0}}(x) \in \mathbb{F}_{q}[x]$ be the minimal polynomial of $\eta^{u_{j_{0}}}$ over $\mathbb{F}_{q}$. Let $k_{j_{0}}=\operatorname{deg} m_{j_{0}}(x)$. Note that $b \leq k_{j_{0}}$ by the assumption $b \leq \min \left\{k_{1}, k_{2}, \ldots, b_{\rho}\right\}$. Using (41) we obtain that $e\left(\eta^{u_{j_{0}}}\right)=0$ and hence $m_{j_{0}}(x) \mid e(x)$. However, this is a contradiction as $\operatorname{deg} e(x) \leq b-1<k_{j_{0}}$. This completes the proof of the statement that $\operatorname{dim}_{\mathbb{F}_{q}} L=b$.

For $0 \leq \ell \leq b-1$, let $F_{\ell}$ be the algebraic function field $F_{\ell}=\mathbb{F}_{q^{r}}\left(x, y_{\ell}\right)$ such that $y_{\ell}^{q}-y_{\ell}=f(x)$. Let $g\left(F_{\ell}\right)$ denote the genus of $F_{\ell}$. Using Condition V.3 it follows from [16, Proposition 3.2.8] that $g\left(F_{\ell}\right) \leq \frac{(q-1)\left(u^{*}-1\right)}{2}$. 
Let $F$ be the algebraic function field $F=$ $\mathbb{F}_{q^{r}}\left(x, y_{0}, y_{1}, \ldots, y_{b-1}\right)$, which is the compositum of the function fields $F_{0}, F_{1}, \ldots, F_{b-1}$. Let $g(F)$ denote the genus of $F$. As $\operatorname{dim}_{\mathbb{F}_{q}} L=b$, it follows from [6, Corollary 6.7] (see also [10, Proposition 3.6] and [17, Lemma 2.4 and (3)]) that

$$
g(F) \leq \frac{\left(q^{b}-1\right)\left(u^{*}-1\right)}{2} .
$$

Let $N^{(\mathrm{aff})}(F)$ denote the number of affine rational places of $F$. As in the proof of Theorem IV.4, for the Hamming weight $w_{H}\left(c^{(b)}(f)\right)$ of $c^{(b)}(f)$ we have

$$
w_{H}\left(c^{(b)}(f)\right)=q^{r}-\frac{N^{(\mathrm{aff})}(F)}{q^{b}} .
$$

Moreover, there is only one rational place of $F$ at infinity. Hence using (42) and Serre's improvement on the Hasse-Weil bound [16, Theorem 5.3.1] yields

$$
\left|N^{(\mathrm{aff})}(F)\right| \leq q^{r}+\frac{\left(q^{b}-1\right)\left(u^{*}-1\right)}{2}\left\lfloor 2 q^{r / 2}\right\rfloor .
$$

Combining (43) and (44) we complete the proof.

Remark 10: There is a codeword of $C$ such that the genus bound (42) is tight. Indeed let $f(x)=a_{1} x^{u_{1}}+a_{2} x^{u_{2}}+\cdots+$ $a_{\rho} x^{u_{\rho}} \in \mathbb{F}_{q^{r}}[x] \backslash\{0\}$ such that the coefficient $a^{*}$ corresponding to $x^{u^{*}}$ is nonzero. Then the genus bound in (42) becomes equality. This always holds if $\rho=1$ and we have equality $g(F)=\frac{\left(q^{b}-1\right)\left(u^{*}-1\right)}{2}$ in Remark 2 instead of the inequality in (42).

In the next remark we explain how Theorem V.4 generalizes an important result of Yaakobi et. al., namely [19, Theorem 1], to arbitrary $b$ and arbitrary $q$ for some cyclic codes.

First we recall that an algebraic function field $F$ with full constant field $\mathbb{F}_{q}$ is called a maximal function field if it attains the upper bound of Hasse-Weil inequality. Namely if $N(F)$ denotes the rational places of $F$ and $g(F)$ denotes the genus of $F$, then $F$ is a maximal function field if and only if

$$
N(F)=1+q^{r}+2 g(F) q^{r / 2} .
$$

It is a difficult open problem to characterize all maximal function fields (see, for example, [8], [9], [16]).

For $1 \leq b \leq n-1$, let $d_{b}(C)$ denote the minimum $b$-symbol Hamming weight $w_{b}(c)$ of codewords as $c$ runs through all nonzero elements of $C$. Note that $d_{b}(C)$ is the $b$-symbol Hamming minimum distance of $C$. Similarly let $D_{b}(C)$ denote the maximum $b$-symbol Hamming weight $w_{b}(c)$ of codewords as $c$ runs through all nonzero elements of $C$.

Remark 11: For any fixed $b$, there are cyclic codes satisfying the conditions of Theorem V.4 such that the lower bound on $w_{b}(c)$ of Theorem V.4 is tight. For instance these codes can be constructed using some maximal algebraic function fields as follows. Note that there are various examples of algebraic functions fields $F=\mathbb{F}_{q^{r}}(x, y)$ of the form $A(y)=f(x)$, where $A(y)$ is a given $q$-additive polynomial of degree $q^{b}$ splitting over $\mathbb{F}_{q^{r}}$ and $f(x) \in \mathbb{F}_{q^{r}}[x]$ is a suitable polynomial. For example if we choose $m$ and put $r=2 m$, then for any divisor $u \mid\left(q^{m}+1\right)$ we obtain a maximal function field as a subcover of the Hermitian function field $H=$ $\mathbb{F}_{q^{2 m}}(x, y)$ given by $y^{q^{m}}+y=x^{q^{m+1}}$. We refer, for example, to [1], [8], [9], for the details. Hence if $u_{1}, u_{2}, \ldots, u_{\rho}$ are ${ }_{1047}$ chosen so that $u^{*}$ becomes a divisor of $\left(q^{m}+1\right)$, then there ${ }_{1048}$ is a codeword of $C$ corresponding to a maximal function field 1049 of the form $A(y)=a_{1} x^{u_{1}}+a_{2} x^{u_{2}}+\cdots+a_{\rho} x^{u_{\rho}}$ with full ${ }_{1050}$ constant field $\mathbb{F}_{q^{r}}$ for some coefficients $a_{1}, \ldots, a_{\rho} \in \mathbb{F}_{q^{r}}$, not ${ }^{1051}$ all zero. This implies that the lower bound of Theorem V.4 is ${ }_{1052}$ tight.

For a given $1 \leq b<\min \left\{k_{1}, k_{2}, \ldots, k_{\mu}\right\}$, let $C$ be a cyclic code such that the lower bound of Theorem V.4 is tight for $b$. Then for the minimum distance $d_{b}(C)$ of $C$ we have

$$
d_{b}(C)=q^{r}-q^{r-b}-\frac{q^{b}-1}{q^{b}}\left(u^{*}-1\right) q^{r / 2} .
$$

For $\delta \geq 1$ and assume that $b+\delta \leq \min \left\{k_{1}, k_{2}, \ldots, k_{\rho}\right\}$ and hence we are in the range for application of Theorem V.4. For $(b+\delta)$-symbol minimum distance $d_{b+\delta}(C)$ using Theorem V.4 we obtain

$$
d_{b+\delta}(C) \geq q^{r}-q^{r-b-\delta}-\frac{\left(q^{b+\delta}-1\right)}{q^{b+\delta}}\left(u^{*}-1\right) q^{r / 2} .
$$

Using (45) and (46) we obtain that

$$
d_{b+\delta}(C) \geq \frac{\left(q^{b+\delta}-1\right)}{\left(q^{b}-1\right) q^{\delta}} d_{b}(C) .
$$

For $q=2$ and $b=\delta=1$, then the inequality in (47) coincides with [19, Theorem 1], which holds for arbitrary binary cyclic codes of dimension at least 2 . We have many further inequalities in (47) for various values of $b, \delta$ and $q$. For $q=2$ and some small values of $b$ and $\delta$, the inequality in (47) gives

$$
d_{2}(C) \geq \frac{3}{2} d_{1}(C), \quad d_{3}(C) \geq \frac{7}{4} d_{1}(C), \quad d_{3}(C) \geq \frac{7}{6} d_{2}(C) .
$$

Here if $q=2$, and $b=\delta=1$, then we get the constant $3 / 2$ above, which corresponds to [19, Theorem 1]. For $q=3$ and some small values of $b$ and $\delta$, the inequality in (47) gives

$d_{2}(C) \geq \frac{4}{3} d_{1}(C), \quad d_{3}(C) \geq \frac{13}{9} d_{1}(C), \quad d_{3}(C) \geq \frac{13}{12} d_{2}(C)$.

In the next corollary we show that if $d_{b}(C)$ is tight for some $1<b \leq \min \left\{k_{1}, k_{2}, \ldots, k_{\rho}\right\}$ in Theorem V.4, then all $d_{\ell}(C)$ are tight for $1 \leq \ell \leq b$. Note that there exist $C$ and $b$ such that $d_{b}(C)$ is tight (see Remark 11).

Corollary 5: We keep the notation and assumptions of Theorem V.4. Assume that there exists an integer $b$ such that $1<b \leq \min \left\{k_{1}, k_{2}, \ldots, k_{\rho}\right\}$ such that

$$
d_{b}(C)=q^{r}-q^{r-b}-\frac{\left(q^{b}-1\right)\left(u^{*}-1\right) q^{r / 2}}{q^{b}} .
$$

Then for any integer $1 \leq \ell \leq b$ we have

$$
d_{\ell}(C)=q^{r}-q^{r-\ell}-\frac{\left(q^{\ell}-1\right)\left(u^{*}-1\right) q^{r / 2}}{q^{\ell}} .
$$

Proof: It follows from the proof of Theorem V.4, there exists $f(x) \in P\left(\left\{u_{1}, u_{2}, \ldots, u_{\rho}\right\}\right)$ such that the function field $F=\mathbb{F}_{q^{r}}\left(x, y_{0}, y_{1}, \ldots, y_{b-1}\right)$, where $y_{i}^{q}-y_{i}=f^{(i)}(x)$ for $1 \leq$ $i \leq b-1$, is a maximal function field. For $1 \leq \ell \leq b-1$, let $F_{\ell}$ be the subfield of $F$ defined as $F_{\ell}=\mathbb{F}_{q^{r}}\left(x, y_{0}, y_{1}, \ldots, y_{\ell-1}\right)$. It is well known that subcovers of maximal function fields are maximal as well [14]. Hence $F_{\ell}$ is a maximal function field (of 
a different genus in general). The proof of Theorem V.2 (see also Remark 11) implies that its bound on $d_{\ell}(C)$ is tight.

Remark 12: Note that in Corollary 5, if the equality on $d_{b}(C)$ holds for some $1<b \leq \min \left\{k_{1}, \ldots, k_{\rho}\right\}$, then all equalities on the minimum distances $d_{\ell}(C)$ hold and these values decrease as $\ell$ decreases. However, in the other direction there is a natural bound by Theorem V.2 and it is important to assume that $b+\delta \leq \min \left\{k_{1}, \ldots, k_{\rho}\right\}$. Indeed if the bound of Theorem V.2 on $d_{b}(C)$ is tight for an integer $1 \leq b \leq$ $\min \left\{k_{1}, k_{2}, \ldots, k_{\rho}\right\}$, then $d_{b+\delta} \geq \frac{\left(q^{b+\delta}-1\right)}{\left(q^{b}-1\right) q^{\delta}} d_{b}(C)$ if $b+\delta \leq$ $\min \left\{k_{1}, k_{2}, \ldots, k_{\rho}\right\}$. However, it follows from Theorem V.2 that $d_{b+\delta+1}(C)=d_{b+\delta}(C)$ if $b+\delta \geq k_{1}+k_{2} \ldots+k_{\rho}$.

We also recall that an algebraic function field $F$ with full constant field $\mathbb{F}_{q}$ is called a minimal function field if it attains the lower bound of Hasse-Weil inequality. Namely if $N(F)$ denotes the rational places of $F$ and $g(F)$ denotes the genus of $F$, then $F$ is a minimal function field if and only if

$$
N(F)=1+q^{r}-2 g(F) q^{r / 2} .
$$

Again characterization of all minimal function fields is a difficult open problem and we have minimal functions fields in the form of maximum function fields mentioned above. Therefore considering minimal function fields instead of maximal function fields we have analogous results of Remark 11 and Corollary 5 on the maximum distances $D_{b}(C)$.

Remark 13: For any fixed $b$, there are cyclic codes satisfying the conditions of Theorem V.4 such that the upper bound on $w_{b}(c)$ of Theorem V.4 is tight. For existence we use similar arguments as in Remark 11 and minimal algebraic function fields instead of maximal algebraic function fields.

For a given $1 \leq b<\min \left\{k_{1}, k_{2}, \ldots, k_{\mu}\right\}$, let $C$ be a cyclic code such that the upper bound of Theorem V.4 is tight for $b$. Then for the maximal distance $D_{b}(C)$ of $C$ we have

$$
D_{b}(C)=q^{r}-q^{r-b}+\frac{q^{b}-1}{q^{b}}\left(u^{*}-1\right) q^{r / 2} .
$$

For $\delta \geq 1$ and assume that $b+\delta \leq \min \left\{k_{1}, k_{2}, \cdots, k_{\rho}\right\}$. For $(b+\delta)$-symbol minimum distance $d_{b+\delta}(C)$ using Theorem V.4 we obtain

$$
D_{b+\delta}(C) \leq q^{r}-q^{r-b-\delta}+\frac{\left(q^{b+\delta}-1\right)}{q^{b+\delta}}\left(u^{*}-1\right) q^{r / 2} .
$$

Using (48) and (49) yield

$$
D_{b+\delta}(C) \leq \frac{\left(q^{b+\delta}-1\right)}{\left(q^{b}-1\right) q^{\delta}} D_{b}(C) .
$$

We present the next corollary on maximum distances, which is an analog of Corollary 5. Its proof follows using similar arguments together with minimal function fields instead of maximal function fields. Note that it is also well known that a subcover of a minimal function field is minimal [14].

Corollary 6: We keep the notation and assumptions of Theorem V.4. Assume that there exists an integer $b$ such that $1<b \leq \min \left\{k_{1}, k_{2}, \ldots, k_{\rho}\right\}$ such that

$$
D_{b}(C)=q^{r}-q^{r-b}+\frac{\left(q^{b}-1\right)\left(u^{*}-1\right) q^{r / 2}}{q^{b}} .
$$

Then for any integer $1 \leq \ell \leq b$ we have

$$
D_{\ell}(C)=q^{r}-q^{r-\ell}+\frac{\left(q^{\ell}-1\right)\left(u^{*}-1\right) q^{r / 2}}{q^{\ell}} .
$$

We can assume that

$$
k_{1} \leq k_{2} \leq \cdots \leq k_{\rho}
$$

without loss of generality. It follows from Theorem V.2 that there is no need to consider $b$-symbol weights if $k_{1}+k_{2} \cdots+$ $k_{\rho}<b \leq n-1$. Hence there are exactly $\rho+1$ regions given below to consider for the full $b$-symbol weight profile of $C$ :

$$
\begin{array}{ll}
\text { Region } 0: & 1 \leq b \leq k_{1}, \\
\text { Region 1: } & k_{1}<b \leq k_{2},
\end{array}
$$

\section{Region $\rho-1: \quad k_{\rho-1}<b \leq k_{\rho}$,}

Region $\rho: \quad k_{\rho}<b \leq k_{1}+k_{2}+\cdots+k_{\rho}$.

It follows from (50) that Region 0 corresponds to Theorem V.4. Next we consider the remaining $\rho$ regions. We need the following notation in order to present our results for the remaining regions neatly. For integers $b, u \in \bar{N}$, let $L, U$ : $\bar{N} \times \bar{N} \rightarrow \bar{N}$ be the functions defined as

$$
\begin{aligned}
& L(b, u)=q^{r}-q^{r-b}-\left\lfloor\frac{\left(q^{b}-1\right)\left(u^{*}-1\right)\left\lfloor 2 q^{r / 2}\right\rfloor}{2 q^{b}}\right\rfloor, \\
& \text { and } \\
& U(b, u)=q^{r}-q^{r-b}+\left\lfloor\frac{\left(q^{b}-1\right)\left(u^{*}-1\right)\left\lfloor 2 q^{r / 2}\right\rfloor}{2 q^{b}}\right\rfloor .
\end{aligned}
$$$$
\text { and }
$$

1150 1151 1152 1153 1154

Note that the functions $L$ and $U$ depend also on $q$ and $r$, which we consider to be fixed. Moreover, these functions correspond to the lower and upper bounds of Theorem V.4. It is easy to observe that as the second parameter $u$ increases (and the first parameter $b$ is fixed), $L(b, u)$ is a decreasing function and $U(b, u)$ is an increasing function.

We are ready to present our bounds for Region 1 in the next theorem.

Theorem V.5: We keep the notation and assumptions of Theorem V.4. We also assume that (50) holds without loss of generality. Recall that $u^{*}=\max \left\{u_{1}, \ldots, u_{\rho}\right\}$ and $w_{b}(c)$ denotes $b$-symbol Hamming weight of a nonzero codeword $c$ of $C$. If $b$ is an integer in Region 1, i.e. $k_{1}<b \leq k_{2}$, then we have

$$
\begin{aligned}
& \min \left\{L\left(b, u^{*}\right), L\left(k_{1}, u_{1}\right)\right\} \\
\leq & w_{b}(c) \\
\leq & \max \left\{U\left(b, u^{*}\right), U\left(k_{1}, u_{1}\right)\right\}
\end{aligned}
$$

Proof: Let $f(x)$ be an arbitrary nonzero polynomial in $P\left(\left\{u_{1}, u_{2}, \ldots, y_{\rho}\right\}\right)$. Let $f_{1}(x)$ and $g(x)$ be the uniquely determined polynomials in $P\left(\left\{u_{1}, u_{2}, \ldots, y_{\rho}\right\}\right)$ such that $f_{1}(x)=a_{1} x^{u_{1}}, g(x)=a_{2} x^{u_{2}}+\cdots+a_{\rho} x^{u_{\rho}}$ and $f(x)=$ $f_{1}(x)+g(x)$. At least one of the polynomials $f_{1}(x)$ and $g(x)$ is nonzero.

If $g(x) \neq 0$, then, as $b \leq k_{2}=\min \left\{k_{2}, k_{3}, \ldots, k_{\rho}\right\}$, we have

$$
\operatorname{dim}_{\mathbb{F}_{q}} \operatorname{Span}\left\{f(x), f^{(1)}(x), \ldots, f^{(b-1)}(x)\right\}=b .
$$

1158 1159 1160 
Moreover, using the methods of the proof of Theorem V.4 we obtain that

$$
L\left(b, u^{*}\right) \leq w_{b}(c) \leq U\left(b, u^{*}\right) .
$$

If $g(x)=0$ and $f_{1}(x) \neq 0$, then it follows from Theorem V.2 that $w_{b}(c)=w_{k_{1}}(c)$ as $k_{1}<b$. Using Theorem V.4 for $k_{1}$, we obtain that

$$
L\left(k_{1}, u_{1}\right) \leq w_{b}(c) \leq U\left(k_{1}, u_{1}\right) .
$$

Combining (52) and (53) we complete the proof.

These methods apply for all regions in (51). It becomes more complicated to state these bounds for Region $j$ as $j$ increases. Next we consider Region 2.

Theorem V.6: We keep the notation and assumptions of Theorem V.4. We also assume that (50) holds without loss of generality. Recall that $u^{*}=\max \left\{u_{1}, \ldots, u_{\rho}\right\}$ and $w_{b}(c)$ denotes $b$-symbol Hamming weight of a nonzero codeword $c$ of $C$. Let $b$ be an integer in Region 2, i.e. $k_{2}<b \leq k_{3}$. Our bounds in this region is presented depending on two cases as follows:

Case $b \leq k_{1}+k_{2}$ : We have

$$
\begin{aligned}
& \min \left\{L\left(b, u^{*}\right), L\left(b, \max \left\{u_{1}, u_{2}\right\}\right), L\left(k_{2}, u_{1}\right), L\left(k_{1}, u_{1}\right)\right\} \\
& \leq w_{b}(c) \leq \\
& \max \left\{U\left(b, u^{*}\right), U\left(b, \max \left\{u_{1}, u_{2}\right\}\right), U\left(k_{2}, u_{1}\right), U\left(k_{1}, u_{1}\right)\right\} .
\end{aligned}
$$

Case $k_{1}+k_{2}<b$ : We have

$$
\begin{aligned}
& \min \left\{L\left(b, u^{*}\right), L\left(k_{1}+k_{2}, \max \left\{u_{1}, u_{2}\right\}\right),\right. \\
& \left.L\left(k_{2}, u_{2}\right), L\left(k_{1}, u_{1}\right)\right\} \leq w_{b}(c) \leq \max \left\{U\left(b, u^{*}\right),\right. \\
& \left.U\left(k_{1}+k_{2}, \max \left\{u_{1}, u_{2}\right\}\right), U\left(k_{2}, u_{2}\right), U\left(k_{1}, u_{1}\right)\right\} .
\end{aligned}
$$

Proof: Let $f_{1}(x)=a_{1} x^{u_{1}}, f_{2}(x)=a_{2} x^{u_{2}}$ and $g(x)=$ $a_{3} x^{u_{3}}+\cdots+a_{\rho} x^{u_{\rho}}$ be the polynomials with coefficients from $\mathbb{F}_{q^{r}}$ so that their sum $f(x)=f_{1}(x)+f_{2}(x)+g(x)$ is not the zero polynomial.

If $g(x) \neq 0$, then we have that $\operatorname{dim}_{\mathbb{F}_{q}}$ Span $\left\{f(x), f^{(1)}(x), \ldots, f^{(b-1)}(x)\right\}=b$ as $b \leq \min \left\{k_{3}, \ldots, k_{\rho}\right\}$. Using Theorem V.4 we obtain that

$$
L\left(b, u^{*}\right) \leq w_{b}(c) \leq U\left(b, u^{*}\right) .
$$

Assume that $g(x)=0$ and $b \leq k_{1}+k_{2}$. If $a_{2} \neq 0$, then using Theorem V.2 and considering the subcases $a_{1} \neq 0$ and $a_{1}=0$ we obtain

$$
\begin{aligned}
& \min \left\{L\left(b, \max \left\{u_{1}, u_{2}\right\}\right), L\left(k_{2}, u_{2}\right)\right\} \\
& \leq w_{b}(c) \leq \max \left\{U\left(b, \max \left\{u_{1}, u_{2}\right\}\right), U\left(k_{2}, u_{2}\right)\right\}
\end{aligned}
$$

If $a_{2}=0$, then $a_{1} \neq 0$ and using Theorem V.2 we get

$$
L\left(k_{1}, u_{1}\right) \leq w_{b}(c) \leq U\left(k_{1}, u_{1}\right) .
$$

Combining (54), (55) and (56) we complete the proof of the case $b \leq k_{1}+k_{2}$.

Next we assume that $g(x)=0$ and $k_{1}+k_{2}<b$. If $a_{1} \neq 0$ and $a_{2} \neq 0$, then using Theorem V.2 we get

$$
\begin{gathered}
L\left(k_{1}+k_{2}, \max \left\{u_{1}, u_{2}\right\}\right) \leq w_{b}(c) \\
\quad \leq U\left(k_{1}+k_{2}, \max \left\{u_{1}, u_{2}\right\}\right)
\end{gathered}
$$

If $a_{2} \neq 0$ and $a_{1}=0$, then similarly we have

$$
L\left(k_{2}, u_{2}\right) \leq w_{b}(c) \leq U\left(k_{2}, u_{2}\right) .
$$

Finally if $a_{2}=0$ and $a_{1} \neq 0$, then we have

$$
L\left(k_{1}, u_{1}\right) \leq w_{b}(c) \leq U\left(k_{1}, u_{1}\right) .
$$

Combining (54), (57), (58) and (59) we complete the proof of the case $b<k_{1}+k_{2}$.

Example 7: Let $q=2, r=12, n=4095, \rho=2$, $u_{1}=3$ and $u_{2}=5$ under notation of Theorem V.6. Using Theorem V.6 we obtain that

$$
\begin{aligned}
& 2880 \leq w_{2}(c) \leq 3264, \\
& 3360 \leq w_{3}(c) \leq 3808, \\
& 3600 \leq w_{4}(c) \leq 4080 .
\end{aligned}
$$

Theorems V.4, V.5 and V.6 present a method to obtain explicit formulas for the bounds on $w_{b}(c)$ for Region $i$ with $i \geq 3$ in (51). It is clear that presenting explicit formulas like in these theorems becomes more involved as the region number $i$ increases. We refrain ourselves from presenting explicit formulas for Region $i$ if $3 \leq i \leq \rho$ as they just use the same ideas and only become more complicated to state. Nevertheless the proofs of Theorems V.2, V.4, V.5 and V.6 give a method to derive lower and upper bounds on the $b$-symbol Hamming weights of arbitrary nonzero codewords of $C$ using algebraic curves. Hence we solve this problem for all regions in (51) implicitly. For any practical situation, and Region $i$ with $3 \leq i \leq \rho$, the methods of this section would be enough to obtain explicit formulas as in Theorems V.4, V.5 and V.6.

Next we extend all of our previous bounds in this section to cyclic codes of length $n \mid\left(q^{r}-1\right)$. Let $C$ be a cyclic code of length $n \mid\left(q^{r}-1\right)$ over $\mathbb{F}_{q}$. Let $U_{0}=\left\{u_{1}, u_{2}, \ldots, u_{\rho}\right\}$ be a basic nonzero set of $C$. Assume that $U_{0} \neq\{0\}$ and each element of $U_{0}$ satisfies Condition V.3. Let $N=\frac{q^{r}-1}{n}$. For integers $0 \leq i$ and $0 \leq u \leq n-1$, it is easy to observe that

$$
u q^{i} \equiv u \quad \bmod n \Longleftrightarrow u N q^{i} \equiv u N \quad \bmod \left(q^{r}-1\right) .
$$

Let $\hat{U}=\left\{u_{1} N, u_{2} N, \ldots, u_{\rho} N\right\}$. Using (60) we get that $\hat{U}_{0}$ is a basic nonzero set for a cyclic code $\hat{C}$ of length $n N=q^{r}-1$ over $\mathbb{F}_{q}$. Moreover, each element of $\hat{U}_{0}$ satisfies Condition V.3 for the length $q^{r}-1$. Let $f(x)=a_{1} x^{u_{1}}+$ $a_{2} x^{u_{2}}+\cdots+a_{\rho} x^{u_{\rho}} \in P\left(U_{0}\right)$ be a nonzero polynomial. Let $c(f) \in C$ be the codeword corresponding to $f(x)$. Put $\hat{f}(x)=a_{1} x^{u_{1} N}+a_{2} x^{u_{2} N}+\cdots+a_{\rho} x^{u_{\rho} N} \in P\left(\hat{U}_{0}\right)$. Let $\hat{c}(\hat{f}) \in \hat{C}$ be the codeword corresponding to $\hat{f}(x)$. As in the proof of Theorem IV.4 we conclude that for any integer $1 \leq b \leq n-1$ we have

$$
w_{b}(c(f))=\frac{1}{N} w_{b}(\hat{c}(\hat{f})),
$$

where $w_{b}(c)$ and $w_{b}(\hat{c})$ denote the $b$-symbol Hamming weight of $c(f)$ and $\hat{c}(\hat{f})$, respectively. These arguments yield the following theorem, which generalizes Theorem V.4.

Theorem V.7: Let $n$ be a divisor of $q^{r}-1$. Let $C$ be an arbitrary cyclic code of length $n$ over $\mathbb{F}_{q}$. Let $U_{0}=$ $\left\{u_{1}, u_{2}, \ldots, u_{\rho}\right\}$ be a basic nonzero set of $C$. Assume that $U_{0} \neq\{0\}$ and each element of $U_{0}$ satisfies Condition V.3. 
Put $N=\frac{q^{r}-1}{n}$ and $u^{*}=\max \left\{u_{1}, u_{2}, \ldots, u_{\rho}\right\}$. Let $\eta \in \mathbb{F}_{q^{r}}^{*}$ be a primitive $n$-th of 1 . For $1 \leq j \leq \rho$ let $k_{j}$ be the index $\left[\mathbb{F}_{q}\left(\eta^{u_{j}}\right): \mathbb{F}_{q}\right]$ of the field extension $\mathbb{F}_{q}\left(\eta^{u_{j}}\right) / \mathbb{F}_{q}$. Let $c \in C$ be an arbitrary nonzero codeword. For $1 \leq b \leq$ $\min \left\{k_{1}, k_{2}, \ldots, k_{\rho}\right\}$, let $w_{b}(c)$ denote the $b$-symbol Hamming weight of $c$. We have

$$
\begin{aligned}
& \frac{1}{N}\left(q^{r}-q^{r-b}-\left\lfloor\frac{\left(q^{b}-1\right)\left(u^{*} N-1\right)\left\lfloor 2 q^{r / 2}\right\rfloor}{2 q^{b}}\right\rfloor\right) \\
& \leq w_{b}(c) \leq \\
& \frac{1}{N}\left(q^{r}-q^{r-b}+\left\lfloor\frac{\left(q^{b}-1\right)\left(u^{*} N-1\right)\left\lfloor 2 q^{r / 2}\right\rfloor}{2 q^{b}}\right\rfloor\right) .
\end{aligned}
$$

In the following remark we explain how we obtain our bounds for length $n \mid\left(q^{r}-1\right)$ using our earlier bounds in this section.

Remark 14: Note that the bounds of Theorem V.7 are obtained from the bounds of Theorem V.4 after applying the following two simple operations in order:

i) change $u^{*}$ to $u^{*} N$ and get the numbers $L^{\prime}$ and $U^{\prime}$ in place of the lower bound $L$ and the upper bound $U$ of Theorem V.4,

ii) divide the numbers $L^{\prime}$ and $U^{\prime}$ obtained in step i) by $N=$ $\frac{q^{r}-1}{n}$ in order to get the lower and the upper bounds of Theorem V.7.

This method applies to all our bounds in Theorems V.4, V.5 and V.6 and we obtain explicit lower and upper bounds for Regions 0,1 and 2 in (51) for any $n$ dividing $q^{r}-1$. Also our arguments after Theorem V.6 regarding the remaining regions, Region $i$ with $3 \leq i \leq \rho$, hold for any length $n$ dividing $q^{r}-1$. Therefore we implicitly solve the problem of obtaining formulas on lower and upper bounds of $b$-symbol weights for these regions if $n$ is an arbitrary positive number dividing $q^{r}-1$.

\section{CONCLUSION}

Let $C$ be an arbitrary cyclic code of length $n$ over $\mathbb{F}_{q}$ with $\operatorname{gcd}(n, q)=1$. Let $b$ be an integer with $1 \leq b \leq n-1$. We gave tight lower and upper bounds for $b$-symbol weights of nonzero codewords of $C$ using algebraic curves over finite fields. We obtained a stability theorem for arbitrary cyclic codes so that the weight enumerator of $b$-symbol Hamming weights of $C$ is the same as the weight enumerator of $k$-symbol Hamming weight of $C$ if $k \leq b \leq n-1$. We improved our lower and upper bounds on $b$-symbol weights of codewords of general cyclic codes for some special subclasses of cyclic codes.

There are still many open problems which require further work in this subject. It is a natural open problem to compute $b$-symbol Hamming weight enumerators of cyclic codes. Construction of explicit classes of optimal cyclic codes for prescribed $b$-symbol would also be interesting. Moreover, generalizing our bounds to the repeated root case, i.e. $\operatorname{gcd}(n, q) \neq 1$ is open.

\section{APPENDIX}

In this appendix we provide necessary background on algebraic function fields in order to make the paper self-contained. For further details we refer, for example, ${ }_{1334}$ to [7], [16].

Let $\mathbb{K}$ be a finite field. An algebraic function field $F$ over $\mathbb{K}$ is a finite extension of the rational function field $\mathbb{K}(x)$ such that any element of $F$ that is algebraic over $\mathbb{K}$ is in $\mathbb{K}$. Here $\mathbb{K}$ is called the constant field of $F$. If $[F: \mathbb{K}(x)]=m$, then there exists a polynomial $h(T)=h_{0}+h_{1} T+\cdots+h_{m} T^{m} \in$ $\mathbb{K}(x)[T]$ of degree $m$ such that $F=\mathbb{K}(x, y)$ and the minimal polynomial of $y$ over $\mathbb{K}(x)$ is $h(T)$. We also call $F$ as an algebraic function field without mentioning $\mathbb{K}$ if it is clear that the constant field is $\mathbb{K}$ from the context.

The simplest algebraic function field is $F=\mathbb{K}(x)$, where $[F: \mathbb{K}(x)]=1$.

A valuation ring of $F$ is a ring $\mathcal{O} \subseteq F$ such that

i) $\mathbb{K} \subsetneq \mathcal{O} \subsetneq F$, and

ii) for any $z \in F \backslash\{0\}$ we have that either $z \in F$ or $z^{-1} \in \mathcal{O}$.

Example 8: Assume that $F=\mathbb{K}(x)$, the rational function field. Let $r(x) \in \mathbb{K}[x]$ be an irreducible polynomial. Then the set

$$
\mathcal{O}_{r(x)}=\left\{\frac{a(x)}{b(x)}: a(x), b(x) \in \mathbb{K}[x], r(x) \nmid b(x)\right\}
$$

is a valuation ring of $F$.

Let $\mathcal{O}$ be a valuation ring of $F$. The group of units of $\mathcal{O}$ is $\mathcal{O}^{\times}=\{u \in \mathcal{O}$ : there exists $v \in \mathcal{O}$ such that $u v=1\}$.

It is well known that $\mathcal{O}$ is a local ring, that there exists a unique maximal ideal $P$ of $\mathcal{O}$, which is given by $P=\mathcal{O} \backslash \mathcal{O}^{\times}$.

A place $P$ of $F$ is the maximal ideal of a valuation ring $\mathcal{O}$ of $F$. Conversely the valuation ring $\mathcal{O}$ is also uniquely determined by its place $P$ as follows: $\mathcal{O}=$ $\left\{z \in F \backslash\{0\}: z^{-1} \notin P\right\} \cup\{0\}$. We denote it $\mathcal{O}_{P}$ and call it the valuation ring of $P$.

Let $P$ be a place of $F$. There exists an element $t \in P$ such that $P=t \mathcal{O}$. This elements is not necessarily unique. Such an element is called a local parameter of $P$. As $P$ is the maximal ideal of its valuation ring $\mathcal{O}_{P}$, the quotient ring $F_{P}=\mathcal{O}_{P} / P$ is a field. $F_{p}$ is called the residue field of $P$. It is well known that $F_{p}$ is a finite extension of $\mathbb{K}$ and the extension degree $\left[F_{p}: \mathbb{K}\right]$ is called the degree of $P$. If the degree of $P$ is one, then we also call that $P$ is a rational place.

Example 9: Assume that $\mathbb{K}=\mathbb{F}_{q}$ and $F=\mathbb{F}_{q}(x)$, the rational function field over $\mathbb{F}_{q}$. There are exactly $q+1$ rational places (degree one places) of $F$ and they are given as follows:

i) For $\alpha \in \mathbb{F}_{q}$, let

$$
P_{\alpha}=\left\{\frac{a(x)}{b(x)}: a(\alpha)=0, b(\alpha) \neq 0\right\},
$$

where $a(x)$ and $b(x) \in \mathbb{F}_{q}[x]$. These form $q$ (affine) ${ }_{1378}$ rational places of $F$.

ii) There is one rational place at infinity of $F$. It is defined as

$$
P_{\infty}=\left\{\frac{a(x)}{b(x)}:, \operatorname{deg} a(x)<\operatorname{deg} b(x)\right\},
$$

where $a(x)$ and $b(x) \in \mathbb{F}_{q}[x]$. 
In general, for $m \geq 1$, an arbitrary place of $\mathbb{F}_{q}(x)$ of degree $m$, different from $P_{\infty}$, is obtained as follows. Let $r(x) \in \mathbb{F}_{q}[x]$ be an irreducible polynomial of degree $m$. Then

$$
P_{r(x)}=\left\{\frac{a(x)}{b(x)}: r(x) \mid a(x), r(x) \nmid b(x)\right\},
$$

where $a(x)$ and $b(x) \in \mathbb{F}_{q}[x]$. is a degree $m$ place of $F$. The notation in (61) and (63) coincide for degree one places: $P_{\alpha}=P_{x-\alpha}$ if $\alpha \in \mathbb{F}_{q}$.

Let $P$ be a place of $F$. Let $\mathcal{O}_{P}$ be its valuation ring and $\mathcal{O}_{P}^{\times}$ be the group of units in $\mathcal{O}_{P}$. We choose a local parameter $t$ of $P$. The discrete valuation $v_{P}$ is a map corresponding to $P$, which is defined as

$$
\begin{aligned}
v_{P}: F & \rightarrow \mathbb{Z} \cup\{\infty\} \\
z & \mapsto \begin{cases}n & \text { if there exists } n \in \mathbb{Z} \\
& \text { and } u \in \mathcal{O}_{P}^{\times} \text {such that } z=t^{n} u, \\
\infty & \text { otherwise (or equivalently if } z=0)\end{cases}
\end{aligned}
$$

It is well known that $v_{P}$ is independent from the choice of the local parameter.

Assume that $E$ and $F$ are algebraic function fields with the same full constant field $\mathbb{K}$. Assume further that $E$ is a finite extension of $F$. Let $P$ be a place of $E$. Then $P^{\prime}=P \cap F$ is a place of $F$. Moreover the residue field $F_{P}$ is a finite extension of the residue field $F_{P^{\prime}}$. The extension degree $\left[F_{P}: F_{P^{\prime}}\right]$ is called the inertia degree of $P \mid P^{\prime}$ and its is denoted as $f\left(P \mid P^{\prime}\right)$. In particular $P$ is a rational place of $E$ if and only if $P^{\prime}$ is a rational place of $F$ and $f\left(P \mid P^{\prime}\right)=1$. Moreover there exists an integer $e$ such that

$$
v_{P}(z)=e v_{P^{\prime}}(z) \text { for all } z \in F .
$$

This integer is called the ramification index of $P \mid P^{\prime}$ and it is denoted as $e\left(P \mid P^{\prime}\right)$. Conversely if $Q$ is a place of $F$, then there are a finite number of places $Q_{1}, \ldots, Q_{\ell}$ in E such that $Q_{i} \cap F=Q$ for $1 \leq i \leq \ell$. A fundamental fact is that

$$
\sum_{i=1}^{\ell} e\left(Q_{i} \mid Q\right) f\left(Q_{i} \mid Q\right)=[E: F] .
$$

Let $s \geq 1$ be an integer. Let $F$ be an algebraic function field with full constant field $\mathbb{F}_{q}$. Let $F \cdot \mathbb{F}_{q^{s}}$ be the smallest extension of $F$ containing $\mathbb{F}_{q^{s}}$. Note that for $s=1$ we have $F=F \cdot \mathbb{F}_{q}$. Let $N\left(F \cdot F_{q^{s}}\right)$ denote the number of rational places of $F \cdot \mathbb{F}_{q^{s}}$. The Hasse-Weil bound [16, Theorem 5.2.3] states that there exists a nonnegative integer $g(F)$, which depends only on $\mathrm{F}$, such that for each positive $s$ integer we have

$$
\left|N\left(F \cdot \mathbb{F}_{q^{s}}\right)-\left(q^{s}+1\right)\right| \leq 2 g(F) q^{s / 2} .
$$

The integer $g(F)$ in (64) is called the genus of $F$. The definition of genus using (64) is not very common, which is an arithmetic method of definition. This definition requires the presentation of the Hasse-Weil bound for all constant field extension $F \cdot \mathbb{F}_{q^{s}}$ with $s \geq 1$. When we state the Hasse-Weil bound, we usually refer to the version of (64) with $s=1$ only. Alternative definitions of genus would require further background like Riemann-Roch Theorem and ramification theory, which we do not need in this paper.

There is an improvement of the Hasse-Weil bound, which is Serre's improvement (see [16, Theorem 5.3.1]). It states that if $F$ is an algebraic function field with full constant field $\mathbb{F}_{q}, \quad{ }_{1432}$ then

$$
|N(F)-(q+1)| \leq g(F)\left\lfloor 2 q^{1 / 2}\right\rfloor .
$$

Let $F$ be an algebraic function field with full constant field $\mathbb{F}_{q}$. Assume that $F$ is an extension of the rational function field $\mathbb{F}_{q}(x)$. Let $P$ be a rational place of $F$. Recall that $\mathbb{F}_{q}(x)$ has exactly $q+1$ rational places. The affine rational places of $\mathbb{F}_{q}(x)$ are $P_{\alpha}$; where $\alpha \in \mathbb{F}_{q}$, and $P_{\alpha}$ defined in (61) in Example 9 above.

In general we call that $P$ is an affine rational place of $F$ if $P \cap F=P_{\alpha}$ for an $\alpha \in \mathbb{F}_{q}$. Otherwise we call that $P$ is a place of $F$ at infinity.

Example 10: Let $r \geq 2$ be an integer. Let $a(x) \in \mathbb{F}_{q^{r}}[x]$ be a polynomial of degree coprime to $q$. Let $=\mathbb{F}_{q^{r}}(x)[y] /<$ $y^{q}-y-a(x)>$. Then $F / \mathbb{F}_{q^{r}}(x)$ is a field extension of degree $q$ and the full constant field of $F$ is $\mathbb{F}_{q^{r}}$.

As in Example 9, for $\alpha \in \mathbb{F}_{q^{r}}$, let $P_{\alpha}$ be an affine rational place of $\mathbb{F}_{q^{r}}$, which corresponds to the irreducible polynomial $x-\alpha \in \mathbb{F}_{q^{r}}[x]$. Let $P_{\infty}$ denote the remaining rational place of $\mathbb{F}_{q^{r}}(x)$, which corresponds to the pole of $x \in \mathbb{F}_{q^{r}}(x)$.

The following characterization of all rational places of $F$ is known. Recall that $\operatorname{Tr}: \mathbb{F}_{q^{r}} \rightarrow \mathbb{F}_{q}$ is the trace map $x \mapsto$ $x+x^{q}+\cdots+x^{q^{r-1}}$. For $\alpha \in \mathbb{F}_{q^{r}}$ and the affine place $P_{\alpha}$ of $\mathbb{F}_{q^{r}}(x)$ we have two cases to consider:

- Case $\operatorname{Tr}(a(\alpha))=0$ : In this case there are exactly $q$ rational places $Q_{\alpha, 1}, Q_{\alpha, 2}, \ldots, Q_{\alpha, q}$ of $F$ such that $Q_{\alpha, i} \cap F=P_{\alpha}$ for each $1 \leq i \leq r$.

- Case $\operatorname{Tr}(a(\alpha)) \neq 0:$ In this case there is no rational place $Q$ of $F$ such that $Q \cap F=P_{\alpha}$.

Moreover there is a unique rational place $Q_{\infty}$ of $F$ such that $Q_{\infty} \cap F=P_{\infty}$.

Let $N^{(\text {aff })}(F)$ denote the number of affine rational places of $F$. These arguments imply that

$$
N^{(\mathrm{aff})}(F)=N(F)-1
$$

and

$$
N^{(\mathrm{aff})}(F)=q\left|\left\{\alpha \in \mathbb{F}_{q^{r}}: \operatorname{Tr}(a(\alpha))\right\}\right|
$$

\section{ACKNOWLEDGMENT}

The authors extend thanks to the anonymous reviewers and the associate editor for their valuable comments and suggestions, which improved the quality and presentation of the manuscript.

\section{REFERENCES}

[1] E. Çakçak and F. Özbudak, "Some Artin-Schreier type function fields over finite fields with prescribed genus and number of rational places," J. Pure Appl. Algebra, vol. 210, no. 1, pp. 113-135, Jul. 2007.

[2] Y. Cassuto and M. Blaum, "Codes for symbol-pair read channels," in Proc. IEEE Int. Symp. Inf. Theory, Austin, TX, USA, Jun. 2010, pp. 988-992.

[3] Y. Cassuto and M. Blaum, "Codes for symbol-pair read channels," IEEE Trans. Inf. Theory, vol. 57, no. 12, pp. 8011-8020, Dec. 2011.

[4] Y. Cassuto and S. Litsyn, "Symbol-pair codes: Algebraic constructions and asymptotic bounds," in Proc. IEEE Int. Symp. Inf. Theory Process., St. Petersburg, Russia, Jul. 2011, pp. 2348-2352. 
[5] C. Ding and J. Yang, "Hamming weights in irreducible cyclic codes," Discrete Math., vol. 313, no. 4, pp. 434-446, Feb. 2013.

[6] I. Duursma, H. Stichtenoth, and C. Voss, "Generalized Hamming weights for duals of $\mathrm{BCH}$ codes, and maximal algebraic function fields," in Arithmetic Geometry and Coding Theory, R. Pellikaan, M. Perret, and S. G. Vlăduț, Eds. 1996 , pp. 53-65.

[7] A. Garcia and H. Stichtenoth, "Algebraic function fields over finite fields with many rational places," IEEE Trans. Inf. Theory, vol. 41, no. 6, pp. 1548-1563, Nov. 1995.

[8] A. Garcia, H. Stichtenoth, and C. Xing, "On subfields of the Hermitian function field," Compos. Math., vol. 120, no. 2, pp. 137-170, 2000.

[9] A. Garcia and F. Özbudak, "Some maximal function fields and additive polynomials," Commun. Algebra, vol. 35, no. 5, pp. 1553-1566, May 2007.

[10] C. Gáneri and F. Özbudak, "Improvements on generalized Hamming weights of some trace codes," Des., Codes Cryptogr., vol. 39, no. 2, pp. 215-231, May 2006.

[11] C. Guneri and F. Ozbudak, "Weil-serre type bounds for cyclic codes," IEEE Trans. Inf. Theory, vol. 54, no. 12, pp. 5381-5395, Dec. 2008.

[12] C. Güneri, F. Özbudak, and F. Özdemir, "Hasse-Weil bound for additive cyclic codes," Des., Codes Cryptogr., vol. 82, nos. 1-2, pp. 249-263, Jan. 2017.

[13] X. Kai, S. Zhu, and P. Li, "A construction of new MDS symbolpair codes," IEEE Trans. Inf. Theory, vol. 61, no. 11, pp. 5828-5834, Nov. 2015.

[14] G. Lachaud, "Sommes d'eisenstein et nombre de points de certaines courbes algébriques sur les corps finis," CR Acad. Sci. Paris, vol. 305, pp. 729-732, Oct. 1987.

[15] F. J. MacWilliams and N. J. A. Sloane, The Theory of Error-Correcting Codes. Amsterdam, The Netherlands: North Holland, 1977.

[16] H. Stichtenoth, Algebraic Function Fields and Codes, vol. 254. New York, NY, USA: Springer, 2009.

[17] G. van der Geer and M. van der Vlugt, "Fibre products of Artin-Schreier curves and generalized Hamming weights of codes," J. Combinat. Theory A, vol. 70, no. 2, pp. 337-348, May 1995.

[18] J. Wolfmann, "New bounds on cyclic codes from algebraic curves," in Coding Theory and Applications (Lecture Notes in Computer Science), vol. 70. Toulon, France: Springer, 1988, pp. 47-62.

[19] E. Yaakobi, J. Bruck, and P. H. Siegel, "Constructions and decoding of cyclic codes over $b$-symbol read channels," IEEE Trans. Inf. Theory, vol. 62, no. 4, pp. 1541-1551, Apr. 2016.
Minjia Shi received the B.S. degree in mathematies frem Anqing Normal University, China, in 2004, the M.S. degree in mathematies from the Hefe University of Technology, China, in 2007, and the Ph.D. degree from the Institute of Computer Network Systems, Hefei University of Technology, China, in 2010. From August 2012 to August 2013, he was a Visiting Researcher with the School of Physical and Mathematical Sciences, Nanyang Technological University, Singapore. From July 2016 to August 2016, he was a Visiting Researcher with Telecom Paris Tech, Paris, France. Later, he visited the Sobolev Institute of Mathematics, in 2020. He has been the Sehool-of Mathematieal Seiences, Anhwi-University, China, since 2007, where he has been a Master's Supervisor and an Associate Professor, sinee April 2012, and a Professor since 2017. He has been a Ph.D. Superviser, sinee 2014. He is the author of over 100 journal articles and two books. His research interests include algebraic coding theory and cryptography.

Ferruh Özbudak received the B.S. degree in electrical and electronics engineering and the Ph.D. degree in mathematics from Bilkent University, Ankara, Turkey, in 1993 and 1997, respectively. He is currently a Professor with Middle East Technical University, Ankara. His research interests include algebraic curves, codes, sequences, cryptography, finite fields, and finite rings.

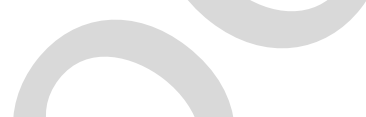

Patrick Solé received the Ingnieur and Docteur-Ingnieur degrees from the Eeole Nationale Stprieure des Tleommmnieations, Paris, France, in 1984 and 1987, respectively, and the Habilitation Diriger Des Recherehes from the Universit de Nice-Sophia Antipolis, Sophia Antipelis, France, in 1993. He has held visiting positions at Syracuse University, Syracuse, NY, USA, from 1987 to 1989 , Macquarie University, Sydney, NSW, Australia, from 1994 to 1996, and Lille University, Lille, France, from 1999 to 2000. Since 1989, he has been a Permanent Member of the CNRS and became a Directeur de Recherche, in 1996. He is currently a member of the CNRS lab I2M, Marseilles, France. He is the author of more than 180 journal articles and four books. His research interests include coding theory (codes over rings, quasi-cyclic codes), interconnection networks (graph spectra, expanders), vector quantization (lattices), and cryptography (Boolean functions, pseudo random sequences). He was a co-recipient of the Best Paper Award for Information Theory, in 1995, given by the Information Theory Chapter of the IEEE. He was an Associate Editor of the IEEE TRANSACTIONS ON INFORMATION THEORY, from 1996 to 1999.

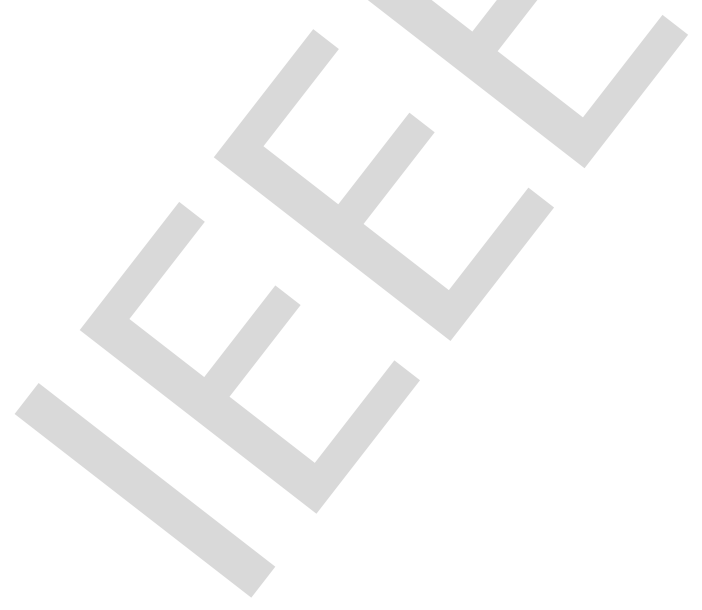

